\title{
Un consenso latinoamericano sobre el síndrome del intestino irritable
}

\author{
J. Valenzuela ${ }^{a}$, J. Alvarado ${ }^{b}$, H. Cohen ${ }^{c}$, A. Damiao ${ }^{d}$, C. Francisconie, L. Frugone ${ }^{f}, J$.C. González ${ }^{g}$, A. Hernández $^{\mathrm{h}}$, \\ B. lade ${ }^{c}$, M.H. Itaqui Lopes ${ }^{e}$, R. Latorre ${ }^{a}, J^{\prime}$ Prado ${ }^{d}$, P. Moraes-Filho ${ }^{d}$, M. Schmulson', L. Soiferi, M.A. Valdovinos', \\ E. Vescok ${ }^{k}$ A. Zalari
}

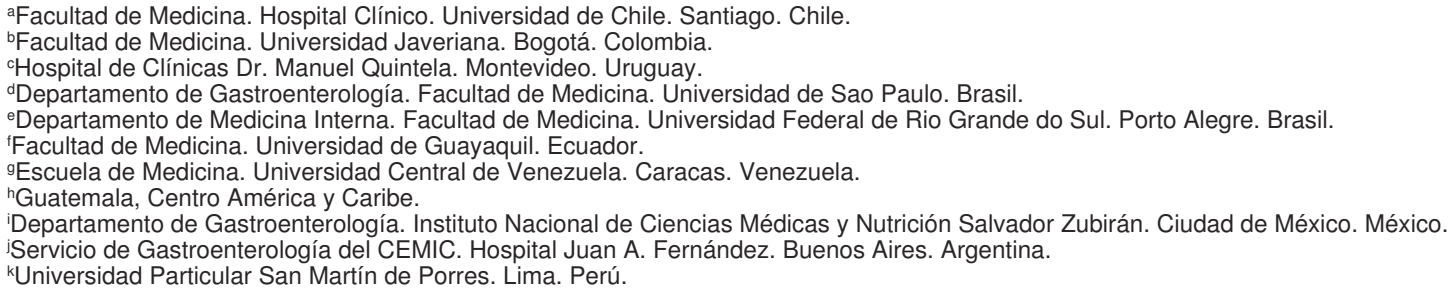

\section{INTRODUCCIÓN}

\section{Generalidades}

El propósito de este artículo es presentar una actualización consensuada de múltiples autores latinoamericanos sobre la prevalencia, fisiopatología, diagnóstico y tratamiento del síndrome del intestino irritable (SII) en adultos. Si bien el SII es un cuadro de alta prevalencia en todo el mundo y se han publicado estudios de diversas regiones ${ }^{1}$, en este artículo se hará hincapié en aspectos que pueden caracterizar la presentación de la enfermedad en la población de pacientes adultos latinoamericanos.

El método utilizado ha sido el siguiente: los autores se reunieron de mayo a noviembre de 2003, se asignaron los temas y hubo discusión hasta llegar a un consenso entre los autores.

Los conceptos que se presentan se basan en evidencias, ya sea de la bibliografía mundial más reciente como de las publicaciones latinoamericanas indexadas pertinentes. Los niveles de evidencia que darán base a los grados de recomendación se señalan en la tabla I.

\section{Definición}

Si bien fueron Manning et $\mathrm{al}^{2}$ los precursores en el uso de un criterio diagnóstico clínico para plantear de forma po-

Este trabajo se llevó a cabo con el apoyo de una beca sin restricciones de Novartis.

Correspondencia: Dr. J. Valenzuela.

Departamento de Gastroenterología.

Hospital Clínico de la Universidad de Chile.

Avda. Santos Dumont, 999. Independencia. Chile.

Correo electrónico: jvalenzu@mi-mail.cl

Recibido el 17-11-2003; aceptado para su publicación el 11-2-2004.
TABLA I. Niveles de evidencia y grados de recomendación ${ }^{1}$

Niveles de evidencia

Nivel I: basados en ensayos aleatorizados, controlados, con valores de $\mathrm{p}<0,05$, con muestras de tamaño suficiente y métodos apropiados

Nivel II: basados en ensayos aleatorizados, controlados, con valores de $\mathrm{p}>0,05$, y ya sea con tamaño insuficiente de la muestra o métodos inapropiados

Nivel III: basados en ensayos no aleatorizados con controles contemporáneos

Nivel IV: basados en ensayos no aleatorizados con controles históricos

Nivel V: basados en estudios de casos

Grados de recomendación

Grado A: basados en evidencias de nivel I

Grado B: apoyados por evidencias de nivel II

Grado C: apoyados por evidencias de niveles III y IV

sitiva el SII, para este trabajo adoptaremos el criterio de SII acordado en la reunión de Roma II ${ }^{3}$, el cual ha llegado a tener aceptación universal. El SII también se conoce en Latinoamérica como «colon irritable», «colon espástico», «colitis nerviosa», «colitis mucosa», entre otras denominaciones.

El SII se caracteriza por malestar o dolor abdominal crónico, recurrente, asociado a alteraciones de la evacuación intestinal, ya sea diarrea, estreñimiento o alternancia en estas alteraciones sin evidencias de daño morfológico o bioquímico en el tubo digestivo y con un examen físico normal. Para satisfacer el criterio de cronicidad, los síntomas deben estar presentes durante 12 semanas en los 12 meses precedentes, aunque no es necesario que sean consecutivas. No debe haber síntomas o signos de alarma como molestias nocturnas que despiertan al paciente, hemorragia rectal, pérdida consistente de peso, fiebre, anemia, anormalidades en el examen físico o de laboratorio habitual. 
TABLA II. Características epidemiológicas de los pacientes con síndrome del intestino irritable de Latinoamérica, EE.UU. y Europa

\begin{tabular}{|l|l|c|}
\hline & $\begin{array}{c}\text { Estados Unidos } \\
\text { y Europa }\end{array}$ & Latinoamérica \\
\hline Prevalencia (\%) & $10-20$ & $9-18$ \\
Edad media (rango), años & $43(20-90)$ & $42(16-89)$ \\
Relación mujeres:varones & $3-4: 1$ & $2-4: 1$ \\
Consultas médicas por año & $2,6-4$ & $3-3,6$ \\
Frecuencia de consulta & & \\
$\quad$ al gastroenterólogo (\%) & 28 & 23 \\
Frecuencia de cirugías abdominales (\%) & $31-37$ & 39 \\
\hline
\end{tabular}

Los pacientes con SII constituyen un grupo heterogéneo $\mathrm{y}$, dado que no existe un marcador biológico que permita establecer el diagnóstico de esta enfermedad, y tampoco alteraciones morfológicas, fisiológicas o bioquímicas características, la definición señalada se basa en la obtención detallada de la historia, el buen juicio clínico y el análisis crítico de los síntomas y signos (recomendación de grado $C$ ), y tiene un valor predictivo positivo de aproximadamente el $98 \%{ }^{4}$.

\section{EPIDEMIOLOGÍA EN LATINOAMÉRICA}

\section{Prevalencia}

El SII es uno de los trastornos de salud más frecuentes en el mundo, con una prevalencia en EE.UU., Europa y Asia del $10-20 \%^{5-11}$ y del $17 \%$ en la población hispana residente en EE.UU. ${ }^{12}$. Por otra parte, la variabilidad en las frecuencias aportadas en la bibliografía se puede deber a diferencias culturales o al uso de criterios diagnósticos diferentes (Manning et $\mathrm{al}^{2}$, Roma I, Roma II), como se demuestra en un reciente estudio europeo multinacional en el que la prevalencia global del SII fue del $11,5 \%{ }^{13}$. Sin embargo, dicha prevalencia resultó del $6,5 \%$ cuando se aplicaron los criterios de Manning et al, del 4,2\%, con los de Roma I y del 2,9\%, con los de Roma II. Según la Asociación Americana de Gastroenterología, el SII constituye el $28 \%$ de las consultas a los gastroenterólogos ${ }^{14}$. En la mayoría de los pacientes, la primera consulta por los síntomas ocurre entre los 30 y los 50 años de edad, y la prevalencia de la enfermedad disminuye después de los 60 años; sin embargo, el SII tiene un amplio rango de edad de presentación ${ }^{9,11-13,15,16}$.

El SII con predominio de estreñimiento es más frecuente en las mujeres que en los varones, con una relación de 3-4 a 1, excepto en la India y Sri Lanka, donde parece ser que predomina en los varones. Esto posiblemente obedece a motivos culturales, ya que los varones tienen un mayor acceso a los servicios de salud en estos países ${ }^{17}$.

En Latinoamérica, en estudios basados en la comunidad, la prevalencia de SII en Brasil, Colombia, México y Uruguay osciló entre el 9 y el $18 \%^{18-23}$. La edad de presentación de los pacientes con SII tiene un amplio rango, de 16 a 89 años, con un promedio de 42 años ${ }^{19,23-26}$. La distribución por sexos del SII en la comunidad es de 2 a 4 mujeres por cada varón ${ }^{20,23-25}$, y afecta aproximadamente a un $15 \%$ de las mujeres y a un $5 \%$ de los varones. Según las encuestas, sólo entre el 10 y el $50 \%$ de las personas que presentan SII consultan al médico; son los denominados «pacientes», en contraposición a los «no pacientes», que son los individuos que cumplen con los criterios diagnósticos del SII, pero que no buscan atención médica. Entre los que buscan atención médica, el 61-71\% son mujeres y entre el 29 y el $39 \%$ son varones ${ }^{19,23,24}$. Aunque sólo una proporción de los pacientes solicita atención médica, los enfermos con SII representan un alto coste a la sociedad, ya que demandan un mayor número de consultas médicas y de estudios paraclínicos; tienen una mayor probabilidad de verse sometidos a cirugías abdominales, especialmen-te apendicectomías, colecistectomías e histerectomías, y presentan mayor absentismo laboral y esco$\operatorname{lar}^{26}$. Estos hallazgos son similares a los comunicados en Europa y EE.UU. ${ }^{11,18}$ (tabla II). En Brasil, el 22\% de los pacientes con SII tiene mayor absentismo laboral, con un promedio de 1-5 días al año de falta de asistencia al trabajo $^{19}$. Hasta el $31 \%$ de los pacientes con SII consulta al gastroenterólogo y este trastorno es el motivo de consulta más frecuente a este especialista ${ }^{22-24}$. Cada paciente demanda un promedio de 3 a 3,6 consultas al año, en comparación con una consulta anual en los pacientes con otros diagnósticos ${ }^{21,25}$. Los pacientes que consultan con mayor frecuencia se ubican en el grupo de edad de 31 a 50 años, de forma similar a lo comunicado en la bibliografía mundial, lo que probablemente guarda relación con el hecho de que éstos presentan síntomas más graves ${ }^{27}$. A pesar de que el diagnóstico se realiza sobre la base de criterios aceptados, se utiliza un alto número de estudios de laboratorio paraclínicos ${ }^{28}$. En México, a los pacientes con SII se les efectúan en promedio 5 estudios de laboratorio y de gabinete antes de llegar al diagnóstico de SII, y se realiza un promedio de 6 estudios anuales posteriormente ${ }^{25}$. Además, los pacientes con SII tienen 22 veces más riesgo de apendicectomía y 2 veces más de colecistectomía ${ }^{26}$.

En Colombia, los pacientes con SII refieren el antecedente de enfermedad similar en el padre y la madre con una frecuencia del 32 y el 20\%, respectivamente, en comparación con el 6 y el $7 \%$ para los controles. Asimismo, el $20 \%$ de los pacientes y el $7 \%$ de los controles comunican el antecedente de «conducta enfermiza» en la infancia ${ }^{20}$. Finalmente, un reciente estudio mejicano en población pediátrica de entre 4 y 16 años de edad encontró una prevalencia del $27 \%$ utilizando los criterios de Roma II ${ }^{29}$.

En resumen, la epidemiología del SII en Latinoamérica no se ha estudiado adecuadamente. Se requieren más estudios en las distintas regiones y países. Éstos deben llevarse a cabo usando los nuevos criterios diagnósticos de Roma II para el SII.

\section{FISIOPATOLOGÍA}

La fisiopatología del SII es extremadamente compleja, porque debemos encontrar explicaciones para síntomas que, al mismo tiempo que carecen de un sustrato orgánico evidente, tienen expresiones clínicas tan opuestas como 


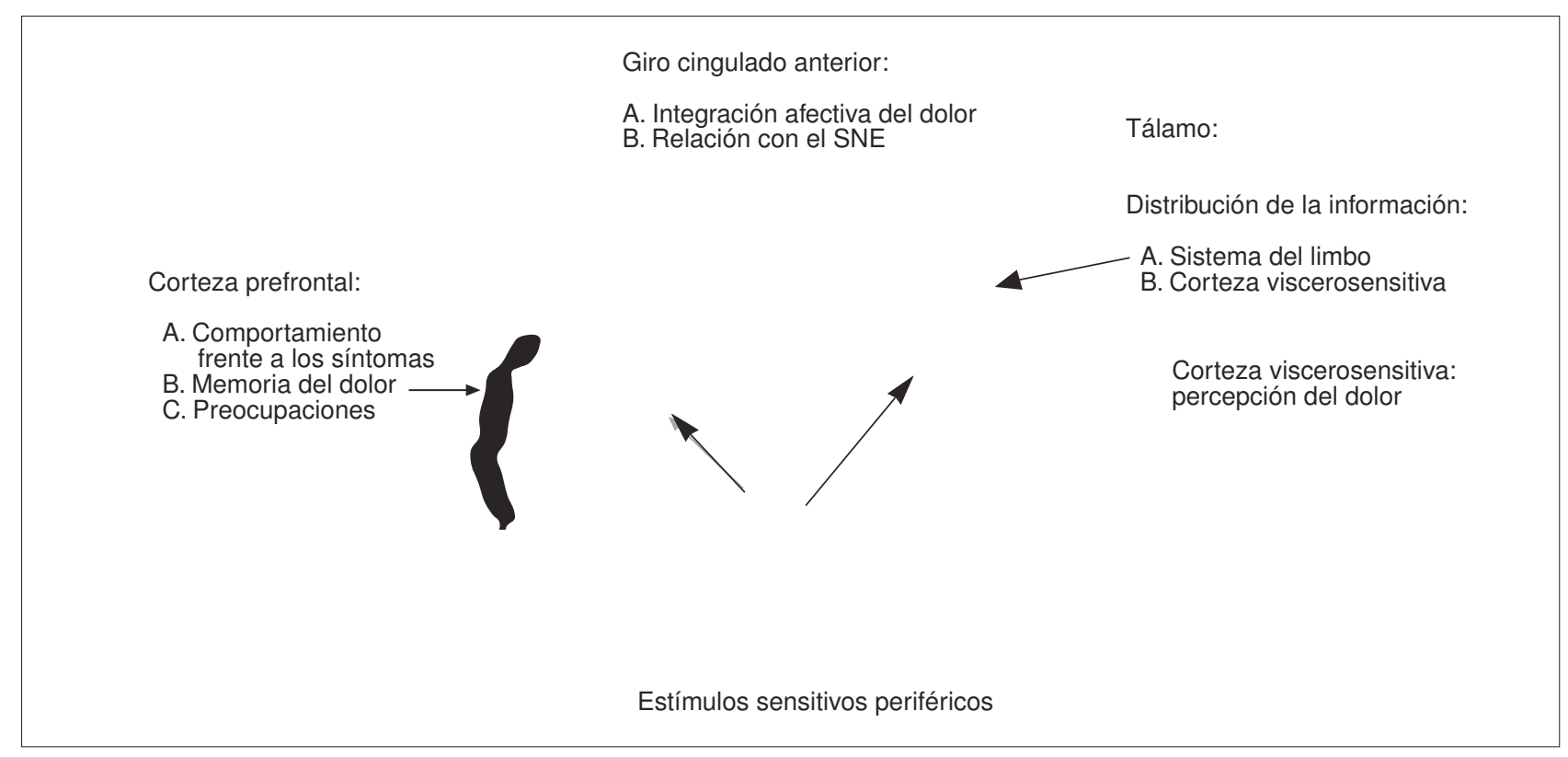

Fig. 1. Áreas del sistema nervioso central relacionadas con la percepción del dolor visceral. SNE: sistema nervioso entérico.

son la diarrea y el estreñimiento. Actualmente, los mecanismos propuestos implican alteraciones en la sensibilidad visceral y en la motilidad intestinal, así como la presencia de factores psicosociales ${ }^{30}$. En la patogenia de este trastorno se ha ido reconociendo progresivamente el relevante papel que desempeñan los mediadores de la inflamación y de la serotonina entérica en el proceso de sensibilidad visceral y en las alteraciones motoras observadas.

\section{Fisiopatología de la hipersensibilidad visceral}

La hipersensibilidad visceral es la disminución del umbral doloroso que se expresa como malestar o incomodidad abdominal. Puede demostrarse experimentalmente inflando pequeños balones dentro del recto o del colon. Los pacientes se quejan de dolor, con volumen y presión menores que los controles normales, y el dolor visceral es de mayor intensidad y más difuso. Es un fenómeno observado en cerca del 50 al $60 \%$ de los pacientes con SII y se interpreta como una alteración en el procesamiento de la sensibilidad. Por otra parte, la sensibilidad a estímulos dolorosos somáticos cutáneos no revela diferencia significativa con los controles ${ }^{31}$.

Los estímulos aferentes sensoriales se transmiten al sistema nervioso central (SNC) por medio de 2 sistemas: el vagosacral y el espinal. El primero conduce predominantemente informaciones sensitivas no dolorosas, mientras que el segundo transmite informaciones predominantemente nociceptivas. Ambas vías transmiten también informaciones eferentes secretomotoras ${ }^{32}$.

Un determinado estímulo nociceptivo periférico, químico o mecánico, estimulará neuronas aferentes que se dirigen al asta dorsal de la médula, llamada primera neurona. En la médula, las dendritas neuronales se ramifican en varios niveles para terminar en más de un nivel medular y hacen sinapsis con una segunda neurona que llevará la información sensitiva a porciones más altas del SNC. Las fibras nerviosas que llevan estas informaciones son los tractos espinotalámico, espinorreticular y la columna dorsal. El tronco cerebral y el tálamo son las estructuras que funcionan como distribuidoras de las informaciones para el cerebro, las que llegan a las áreas de percepción dolorosa viscerosensoriales y áreas que confieren un componente afectivo al estímulo. Hay áreas sensitivas de la corteza cerebral que están relacionadas con la intensidad del dolor, mientras que los centros límbicos ofrecen el resultado final emocional de la experiencia dolorosa (fig. 1). Varios circuitos cerebrales participan simultáneamente en el procesamiento de estas informaciones, y las áreas en el giro cingulado y en el tálamo parecen ser críticas en el procesamiento emocional de la información ${ }^{32}$.

Una vez que el SNC ha percibido el dolor, se emite un estímulo eferente y se realizan sinapsis en el bulbo y en el puente. Esto se traducirá en la liberación de sustancias serotoninérgicas u opiáceas y permitirá que este estímulo se inhiba o amplíe en el asta dorsal de la médula. Estas neuronas también puede liberar noradrenalina, y tanto ésta como la serotonina pueden liberar opiáceos de las neuronas localizadas en el asta dorsal de la médula.

Para que se dé el fenómeno de la hipersensibilidad visceral una posibilidad teórica sería que se produjeran alteraciones en la función en las diferentes áreas de procesamiento de la información sensitiva, es decir, tanto en el sistema nervioso entérico como en la médula o en los centros más altos del SNC.

Las alteraciones de la sensibilidad suceden simultáneamente en los 3 niveles. La expresión «eje cerebro-intestino» (gut-brain axis) se creó para describir esta relación directa entre el SNC y el tubo digestivo: acontecimientos im- 
portantes que se originan en el SNC, a través de la percepción por los órganos de los sentidos y por las emociones, pueden interferir en la función del aparato digestivo, del mismo modo que enfermedades intestinales pueden provocar reacciones relevantes en el SNC (p. ej., depresión o ansiedad provocadas por una infección intestinal o una enfermedad inflamatoria intestinal).

Existen evidencias de que los neurotransmisores que participan en el complejo mecanismo de percepción en la pared intestinal pueden funcionar de manera inadecuada en los pacientes con SII. Entre ellos destacan los distintos receptores de la serotonina, principalmente de los tipos 5-HT3 y 5-HT4, las neurocininas y el péptido relacionado con el gen de la calcitonina, los cuales pueden inhibir dolores agudos periféricos. Otros mediadores como la bradicinina y la histamina probablemente también participan en este proceso. En la médula, el péptido relacionado con el gen de la calcitonina y las neurocininas y, en el SNC, el Nmetil-D-aspartato y la corticotropina también participan en el proceso de percepción de dolor agudo que se puede inhibir con antagonistas específicos. Si estos mecanismos se vuelven más sensibles, como en situaciones con mucosa inflamada (estimulación periférica o medular) y factores de estrés psicosociales, como abuso sexual (estimulación central), se pueden explicar el desencadenamiento y la persistencia de los síntomas en pacientes con SII. La hormona liberadora de la corticotropina aumenta en situaciones de estrés y muy probablemente participa de manera importante en las diferentes áreas del sistema nervioso, dando lugar tanto a hipersensibilidad visceral como a alteraciones motoras en estos pacientes ${ }^{31,33}$.

Estudios que evalúan el flujo sanguíneo como indicador de activación cerebral, a través de imágenes registradas por tomografía por emisión de positrones o por resonancia magnética funcional, señalan que distintas áreas del cerebro se comportan de manera diferente frente a un estímulo doloroso periférico en poblaciones de pacientes con SII si se les compara con controles sanos. Las áreas que se ha demostrado que responden a este tipo de estímulo son el giro cingulado anterior, la corteza de la ínsula, el tálamo y la corteza prefrontal. Los estímulos, tanto si son reales como si tan sólo se preven dichos acontecimientos, estimulan estas áreas de manera diferente en los pacientes con SII. Se observa un aumento de conexiones entre el giro cingulado anterior y el lóbulo prefrontal en pacientes con SII, lo que podría explicar el estado de hipervigilancia observado en algunos de estos pacientes. La activación del giro cingulado anterior se correlaciona con la intensidad del dolor manifestado por los pacientes. También se ha observado que acontecimientos psicosociales como los abusos, por ejemplo, pueden alterar la actividad del giro cingulado anterior ${ }^{33,34}$.

Se ha señalado la influencia del sexo femenino en la percepción de los síntomas del SII ${ }^{35}$. Las mujeres, cuando se las compara con los varones, relatan con mayor frecuencia y mayor variabilidad síntomas gastrointestinales y extraintestinales. Además, el $40 \%$ de las mujeres refiere aumento de los síntomas durante el ciclo menstrual, a pesar de que no existen diferencias en las concentraciones plasmáticas de estradiol y progesterona entre aquéllas con y sin SII.

Un estudio en nuestra población ha demostrado que el chile en la dieta disminuye la tolerancia al dolor en respuesta a la distensión rectal. Esto contrasta con otros estudios que han mostrado un efecto analgésico de dicho componente, y esto probablemente se debe a diferencias en las dosis utilizadas, ya que se sabe que dosis altas de capsaicina producen apoptosis celular y por tanto analgesia, mientras que dosis bajas pueden estimular los receptores de la capsaicina en las terminales nerviosas ${ }^{36}$.

\section{Fisiopatología de las alteraciones de la motilidad intestinal}

Cualitativamente, parece no haber alteraciones motoras específicas del SII. Por lo tanto, resulta difícil entender cómo algunos pacientes presentan una aceleración de tránsito del colon, expresado clínicamente por evacuaciones con diarrea, mientras que en otros éste está disminuido y presentan estreñimiento, y en un tercer grupo se alternan estos 2 síntomas ${ }^{33}$.

La alteración motora más consistentemente observada en pacientes con SII es la presencia de una respuesta motora exacerbada a varios estímulos, tales como ingestión de alimentos, estrés psicológico, distensión del intestino con balón, colecistoquinina, hormona liberadora de corticotropina y fármacos colinérgicos (neostigmina). Los mecanismos involucrados en esta hiperreactividad motora parecen estar relacionados con el funcionamiento de las interrelaciones entre el sistema nervioso entérico y las fibras musculares lisas del tubo digestivo (fig. 2). Algunos pacientes con estreñimiento presentan disminución de las células intersticiales de Cajal en el colon. Estas células tendrían una función de marcapaso en la actividad eléctrica que regula la motilidad del colon. El reflejo gastrocólico exacerbado es un ejemplo de hiperactividad motora desencadenada por la alimentación ${ }^{33}$.

El exceso de gases y la distensión abdominal, referida con mucha frecuencia por los pacientes, probablemente se relacionan con la retención de gas en el intestino más que con un aumento de la producción de gases ${ }^{37}$.

Los otros síntomas —urgencia para evacuar, sensación de evacuación incompleta o dolor relacionado con la actividad motora del colon - son más bien una manifestación de la hipersensibilidad visceral que de una alteración de la motilidad.

\section{Papel de la inflamación en la patogenia del síndrome del intestino irritable}

Aproximadamente entre el 6 y el $30 \%$ de los pacientes con SII señalaron que sus síntomas se habían iniciado después de un episodio indicativo de infección intestinal aguda. Esta observación apoya la hipótesis de que en un subgrupo de pacientes con SII los síntomas pueden ser debidos a alteraciones funcionales relacionadas con el proceso inflamatorio y provocadas por las bacterias patógenas del intestino. Sin embargo, no se han demostrado alteraciones 
Fig. 2. Integración del sistema nervioso central con el sistema nervioso entérico.

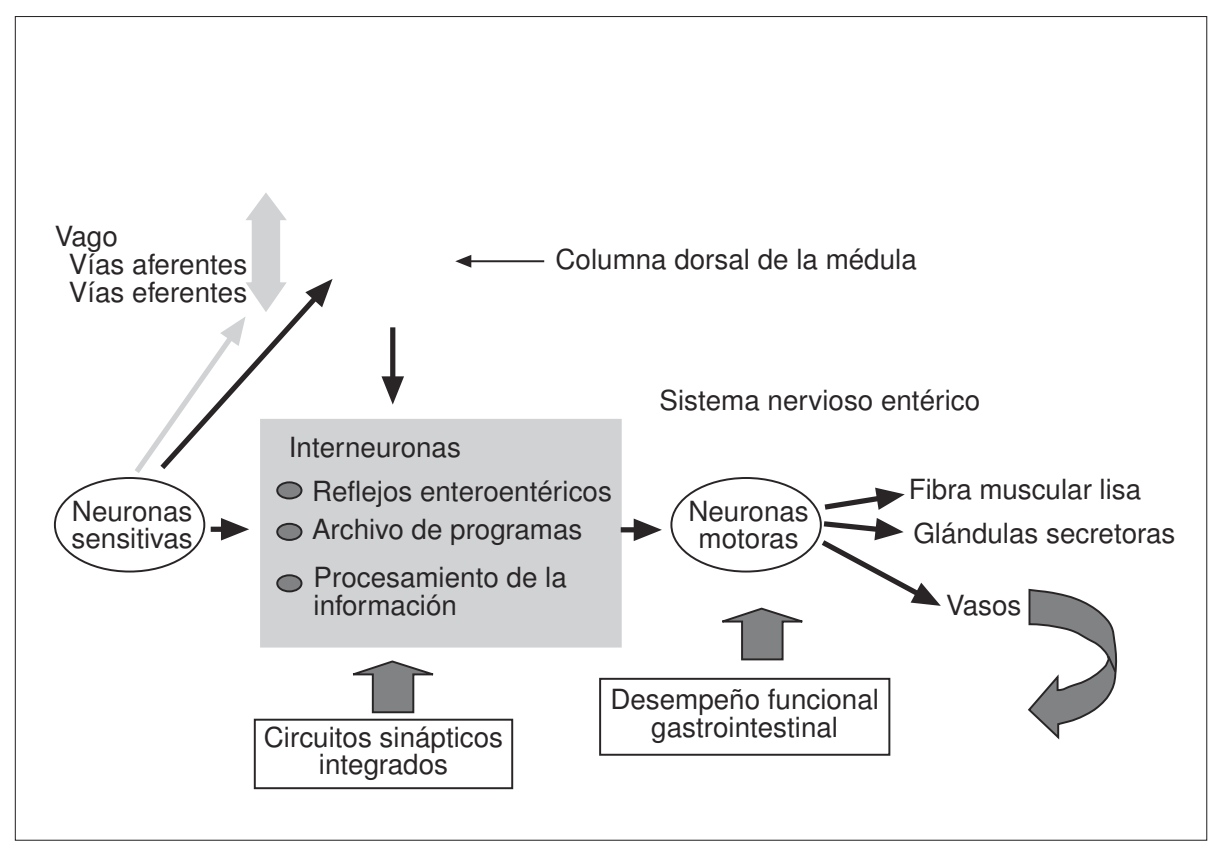

histológicas en las biopsias de colon de estos pacientes. Recientemente, en un grupo de pacientes con cuadro de SII postinfección por Campylobacter enteritis se observó un aumento de células enterocromafines, que producen más 5-HT y CD3 que liberan más citocinas, lo que altera la motilidad y reduce el umbral visceral por un período de hasta un año después del episodio de infección. El resultado final sería la sensibilización de las vías aferentes sensitivas y el aumento de la actividad propulsora y de la secreción de agua y electrólitos hacia la luz intestinal. El aumento de las células enterocromatofines podría tener implicaciones con la producción aumentada de serotonina en la mucosa del colon de estos pacientes ${ }^{38}$. Otros estudios demuestran que acontecimientos psicosociales en el momento que los pacientes presentan un cuadro de diarrea infecciosa tienen una influencia significativa en el desenlace clínico al actuar como factores de perpetuación de los síntomas de SII. Los factores de riesgo para el desarrollo de SII postinfeccioso serían la duración del episodio infeccioso, el sexo femenino, la hipocondriasis, el estrés en el momento del cuadro infeccioso y factores bacterianos mal definidos ${ }^{38}$.

El significado de la infección como acontecimiento que desencadena el SII se desconoce en los países de Latinoamérica. Hay regiones pobres de Brasil en las que la población presenta más de 10 episodios anuales de infección intestinal. Sin embargo, no existe evidencia epidemiológica de que en Brasil el SII sea más frecuente en estas poblaciones, como sería de esperar si del 7 al 30\% de esta población desarrollase SII postinfeccioso.

\section{Papel de los acontecimientos psicosociales}

Se ha propuesto un modelo en que las variables genéticas asociadas a eventos psicosociales tempranos en la vida de las personas pueden tener repercusiones que aparecerán a lo largo de la vida en términos del funcionamiento del aparato digestivo y de susceptibilidad a determinados procesos patológicos, y que a su vez influirían en la experiencia que la persona tenga de su síntoma y en el desenlace clínico de la enfermedad ${ }^{39}$. Las variables que deben considerarse dentro de este modelo son:

1. Experiencias iniciales en la vida: actitudes de compensación o gratificación familiar frente a enfermedades, pérdidas de cualquier naturaleza y maltrato físico o abuso sexual.

2. Refuerzo del comportamiento del enfermo. Los pacientes con SII tienden a tener más síntomas somáticos y utilizan más el sistema de salud por síntomas menores.

3. Antecedente de maltrato físico y abuso sexual. En la población general, en atención primaria, los pacientes con SII tienen 3 veces más antecedentes de abuso sexual. Se han planteado 2 posibilidades para explicar esta relación: a) la situación de abuso desencadena directamente el SII por alteraciones relacionadas con estrés en el SNC, y $b$ ) el antecedente de abuso sería un elemento catalizador de las alteraciones psicológicas y de la hipersensibilidad visceral, ya existentes en el SNC de estos pacientes ${ }^{40,41}$.

4. Relación entre síntomas psiquiátricos y SII. Existe una relación directa entre la gravedad de los síntomas de SII y los síntomas psicológicos (comorbilidad) ${ }^{42,43}$.

\section{CALIDAD DE VIDA. IMPACTO ECONÓMICO}

La calidad de vida es el goce de bienestar físico, mental y social. Por su alta prevalencia, su naturaleza funcional y su carácter crónico, el SII interfiere en la calidad de vida y tiene, por lo tanto, importancia social y económica. Los pacientes con dicha enfermedad presentan frecuentemen- 
TABLA III. Instrumentos de calidad de vida relacionada con la salud utilizados en el síndrome del intestino irritable

\begin{tabular}{|c|c|c|}
\hline Instrumento & Propiedades psicométricas evaluadas & N. ${ }^{\circ}$ de ítems \\
\hline IBSQOL, autoadministrable (específico) & $\begin{array}{l}\text { Funciones emocional, mental, salud, bienestar, sueño, energía, función física, dieta, } \\
\text { papel social y relaciones sexuales }\end{array}$ & 30 \\
\hline $\begin{array}{l}\text { IBS-QOL, entrevistador + autoadministrable } \\
\quad \text { (específico) }\end{array}$ & $\begin{array}{l}\text { Disforia, interferencia con las actividades, imagen corporal, preocupación relacionada } \\
\text { con la salud, preocupación relacionada con los alimentos, reacciones sociales, } \\
\text { relaciones sexuales, índice de síntomas e índice de incomodidad }\end{array}$ & 34 \\
\hline IBSQ (específico) & Síntomas intestinales, fatiga, limitación de actividades, trastorno emocional & 26 \\
\hline $\begin{array}{l}\text { SF-36 (Short form Health Survey), } \\
\text { autoadministrable (genérico) }\end{array}$ & $\begin{array}{l}\text { Función física, dolor corporal, actividad física, actividad social, salud mental, vitalidad } \\
\text { y estado general de salud }\end{array}$ & 36 \\
\hline
\end{tabular}

Modificada de El-Serag ${ }^{51}$.

te perturbaciones de su calidad de vida que dan lugar a trastornos en sus relaciones sociales, alteraciones de orden psicológico y del sueño, déficit funcional en el trabajo, perjuicio en el entretenimiento, preocupaciones con la dieta y limitaciones en la actividad sexual ${ }^{44,45}$.

\section{Evaluación de la calidad de vida}

Existen numerosos instrumentos que evalúan la calidad de vida. Pueden ser de 2 tipos: evaluaciones globales o genéricas, por un lado, y específicas para una enfermedad, por otro. Los instrumentos genéricos miden el impacto de determinadas enfermedades en la vida de los pacientes, en grandes poblaciones. Por el contrario, los instrumentos específicos son más sensibles y pueden evaluar, en bases individuales y específicas, ciertos aspectos de la calidad de vida, motivo por el cual tienen mayor capacidad para detectar la mejoría o el empeoramiento antes y después del tratamiento. En la evaluación de la calidad de vida se consideran las dimensiones emocionales, psicológicas y sociales de las enfermedades, además de las dimensiones físicas. En el caso del SII, se reconoce que la calidad de vida es un importante marcador de este trastorno ${ }^{46,47}$. Los tests, por lo tanto, se utilizan cada vez más por su importancia no sólo meramente académica, sino también por el interés en la práctica médica diaria, como, por ejemplo, en la evaluación de la eficacia de los tratamientos. Así pues, en lo que se refiere al SII, conviene que los gastroenterólogos estén familiarizados con los instrumentos más frecuentes que tienen demostrada consistencia. En la tabla III se presentan los instrumentos genéricos y específicos de enfermedad que se pueden usar para evaluar la calidad de vida en el SII. En general, los estudios son de 3 órdenes: a) aquellos que comparan la calidad de vida en el SII con la de individuos sanos; $b$ ) otros que la comparan con la de pacientes que presentan otras enfermedades, y c) los que incluyen determinantes tales como el sexo, el tipo y la gravedad de los síntomas y la respuesta a un tratamiento.

Hay que recordar que la calidad de vida está muy comprometida en los pacientes con enfermedades gastrointestinales, independientemente del diagnóstico ${ }^{48}$, pero todo hace indicar que los pacientes con quejas funcionales presentan los niveles más bajos cuando se les compara con individuos que presentan otros trastornos, incluyendo aquellos que presentan alta mortalidad, como la insuficiencia cardíaca y la diabetes mellitus tipo $2^{49}$. El ideal del estudio de la calidad de vida es la combinación de métodos genéricos y específicos $^{50}$.

El compromiso de la calidad de vida está relacionado con la gravedad de los síntomas del SII ${ }^{51}$. Este compromiso es comparable al que producen ciertas enfermedades orgánicas, tales como el asma bronquial, la diabetes tipo 2 y el reflujo gastroesofágico ${ }^{52}$. Una respuesta terapéutica satisfactoria del SII lleva aparejada una mejoría de la calidad de vida $^{51}$.

\section{Impacto económico}

Vale la pena comentar que los datos reales que expresan los gastos en salud no son exactos porque muchos estudios se refieren solamente a los centros de referencia especializados a los que se envió a los pacientes, y no necesariamente a los gastos generales derivados de todas las visitas a los centros de atención. De cualquier manera, el impacto económico del SII es bastante grande y hay que considerar los gastos directos e indirectos.

Los gastos directos son los relacionados con el sistema de salud, muy particularmente con la atención médica repetitiva y de curso prolongado. Los pacientes con enfermedades funcionales tienen la costumbre de visitar muchas veces al médico y el éxito del tratamiento depende también de una fuerte relación médico-paciente ${ }^{53}$. Otro coste directo importante está relacionado con los exámenes subsidiarios y con el uso de medicamentos en general, como los antiinfecciosos, antiinflamatorios no esteroideos, analgésicos y antidepresivos, además de los fármacos indicados para el tratamiento de los síntomas de $\mathrm{SII}^{54}$. Los gastos de exámenes adicionales pueden verse incrementados aún más si las sociedades latinoamericanas empiezan a imitar los hábitos de la sociedad estadounidense, donde el temor de los médicos a las demandas por mala práctica aumentan el número de exámenes innecesarios.

Los costes indirectos del SII están relacionados con la disminución de la productividad laboral. Nuevamente, los datos de una encuesta realizada en EE.UU. revelaron que los pacientes con SII tenían un absentismo anual un $40 \%$ mayor que los que no presentan este trastorno. No existen datos comparables en la bibliografía latinoamericana. Más difíciles de calcular son las pérdidas por deterioro funcional y los gastos de transporte, además de los gastos intangibles, como los derivados de los cambios dietéticos que in- 
ciden muchas veces en la pobre calidad de vida que estos pacientes presentan ${ }^{54,55}$. No hay estudios específicos sobre el tema en Latinoamérica.

En resumen, el SII altera la calidad de vida en los pacientes y ocasiona un gasto económico importante. Se requieren más estudios que utilizando métodos validados cuantifiquen el problema en las distintas regiones y países latinoamericanos.

\section{MANIFESTACIONES CLÍNICAS Y DIAGNÓSTICO}

El SII se consideró, hasta hace poco tiempo, un «diagnóstico de exclusión», dado su carácter de síndrome o trastorno funcional caracterizado por la ausencia de daño estructural demostrable o de alteración evidente mediante los métodos habituales de estudio en un paciente con síntomas digestivos. Esta concepción ha cambiado desde la aparición de los diversos «criterios diagnósticos» que buscan una mayor precisión en la categorización de los pacientes y que han hecho de él un «diagnóstico de inclusión», casi similar en sus características operativas a, por ejemplo, la depresión o el síndrome coronario agudo.

La descripción operativa de los criterios diagnósticos se inicia con Manning et al, quienes describieron su criterios en $1978^{56}$, y culmina con la elaboración de los criterios de Roma I y II. Otros investigadores han contribuido a precisar o comprobar la sensibilidad y especificidad de estos criterios en diferentes situaciones y grupos de población ${ }^{56-}$ 58 .

Los criterios de Manning son el resultado de un análisis estadístico de prevalencia y frecuencia de aparición de síntomas en un grupo de pacientes con diagnóstico muy probable de SII, y se centran en la presencia de dolor, distensión y aparición de cambios en las características de la evacuación ${ }^{2}$.

Los criterios de Roma I y posteriormente los de Roma II se obtienen por consenso de expertos y buscan una mayor precisión diagnóstica, dado el rigor en la exclusión de otras patologías orgánicas o funcionales ${ }^{58}$.

\section{Manifestaciones clínicas}

El SII, aunque afecta a un grupo heterogéneo de pacientes, se puede definir como un trastorno gastrointestinal caracterizado por dolor o malestar abdominal crónico, asociado casi siempre a alteración del hábito intestinal, en ausencia de cualquier causa orgánica demostrable; puede afectar a cualquier grupo de edad, parece predominar en mujeres y se asocia con frecuencia a otros trastornos funcionales del aparato digestivo ${ }^{59}$.

Los pacientes con SII pueden consultar por un amplio espectro de síntomas, gastrointestinales o extraintestinales, pero el complejo sindrómico de dolor abdominal, alteración del hábito intestinal y distensión abdominal continúa siendo la piedra angular del diagnóstico, a pesar de su poca especificidad $^{60}$.

\section{Síntomas gastrointestinales}

\section{Dolor abdominal crónico}

Los pacientes pueden describir casi cualquier tipo de dolor o malestar, pero usualmente lo señalan como de tipo cólico o calambre intestinal de intensidad variable y aparición periódica, casi siempre localizado en el abdomen inferior y muy a menudo en el flanco izquierdo o en la llamada «cuerda cólica izquierda ${ }^{61}$. Con frecuencia su inicio se asocia a estrés o comidas y se alivia con la defecación. El dolor es de predominio diurno y desaparece o mejora en la noche, sin generar insomnio de conciliación, y nunca se asocia a anorexia, desnutrición o pérdida de peso. El dolor debe estar siempre presente en los pacientes con diagnóstico de SII.

\section{Alteración del hábito intestinal}

Por definición, al igual que el dolor, los pacientes deben tener alteración del hábito intestinal, en ocasiones muy difícil de precisar dado el rango tan amplio, universalmente aceptado, de normalidad en el número y consistencia de la defecación. Los pacientes pueden quejarse de diarrea, estreñimiento o alternancia de ambos, y su distribución según un estudio europeo ${ }^{62}$ puede ser la siguiente: predominio de estreñimiento en el $47 \%$ de los casos, predominio de diarrea en el $26 \%$ y con alternancia en el $27 \%{ }^{2}$. Por otro lado, el hábito intestinal predominante puede condicionar otros síntomas, como son la distensión y el dolor en el bajo vientre y la sensación de evacuación incompleta ${ }^{46}$.

La diarrea del SII es semiológicamente característica de intestino grueso: frecuente, de volumen pequeño o moderado, posprandial y urgente, y se puede acompañar de moco y sensación de evacuación incompleta. No debe tener sangre. Casi siempre ocurre durante la jornada laboral y especialmente en horas matutinas.

El estreñimiento, que puede durar días o meses o alternarse con episodios de normalidad o diarrea, casi siempre se expresa como la presencia de heces muy duras, caprinas, asociadas a sensación de evacuación incompleta, distensión rectal, aumento del esfuerzo defecatorio, tiempo de evacuación prolongado y uso crónico de enemas o laxantes.

\section{Otros síntomas}

Otros síntomas digestivos predominantes son la distensión abdominal, la eliminación de moco por el recto y la sensación de evacuación incompleta. Tienen una frecuencia de aparición menor y no son claves para el diagnóstico basado en criterios del SII.

\section{Síntomas extradigestivos}

La frecuencia de aparición de síntomas o de entidades extradigestivos, asociados o no realmente al SII (relación de causalidad o relación de asociación no causal), es muy 
TABLA IV. Síndrome del intestino irritable: criterios de Manning et $\mathrm{al}^{2}$

Cuatro síntomas estadísticamente más frecuentes en el síndrome del intestino irritable:

Evacuación asociada al inicio del dolor

Borborismo asociado al inicio del dolor

Dolor que mejora con la deposición

Distensión abdominal visible

Dos síntomas frecuentes:

Mucorrea

Sensación de evacuación incompleta

\section{TABLA V. Criterios de Roma II}

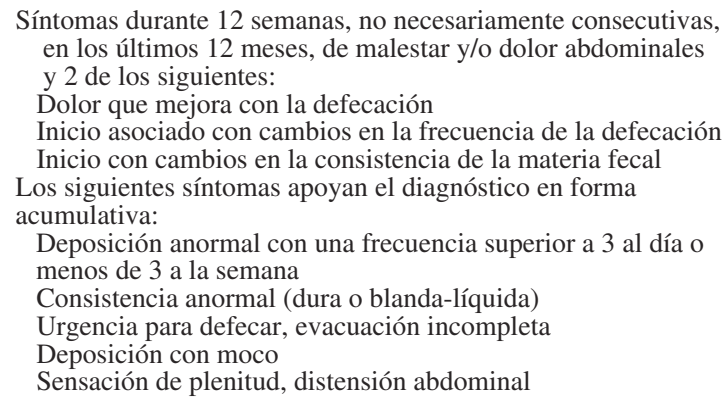

Síntomas durante 12 semanas, no necesariamente consecutivas, en los últimos 12 meses, de malestar y/o dolor abdominales y 2 de los siguientes:

Dolor que mejora con la defecación

Inicio asociado con cambios en la frecuencia de la defecación

Inicio con cambios en la consistencia de la materia fecal

Los siguientes síntomas apoyan el diagnóstico en forma

acumulativa:

Deposición anormal con una frecuencia superior a 3 al día o

menos de 3 a la semana

Consistencia anormal (dura o blanda-líquida)

Urgencia para defecar, evacuación incompleta

Deposición con moco

Sensación de plenitud, distensión abdominal

\section{TABLA VI. Signos de alarma en el síndrome del intestino} irritable

Historia: pérdida de peso, inicio en un paciente mayor de 50 años, síntomas nocturnos e historia familiar de cáncer o enfermedad inflamatoria intestinal

Laboratorio: hemoglobina baja, aumento de los leucocitos, aumento de la velocidad de sedimentación o química anormal

Hallazgos físicos: anormalidad en el examen, fiebre y sangre oculta en las deposiciones

amplia y en gran parte se da por la alta frecuencia de aparición del síndrome en la población general. Existe, sin embargo, una asociación demostrada entre el SII y la disfunción sexual, la dispareunia, la dismenorrea, la polaquiuria y el tenesmo vesical, la hipertensión arterial sistémica, la hiperreactividad bronquial y la fibromialgia ${ }^{63-65}$.

\section{Proceso diagnóstico}

La introducción de los criterios de Manning et $\mathrm{al}^{2}$ y de los derivados de las Reuniones de Consenso de Roma I y Roma I $^{58}$ ha permitido tener una mayor precisión diagnóstica y un mayor rigor en el diseño de estudios que buscan medir la eficacia terapéutica de medicamentos en el SII. Los criterios de Manning et $\mathrm{al}^{2}$ (tabla IV), muy útiles en la práctica clínica diaria, son menos específicos y sensibles que los criterios de Roma II (tabla V), pero logran mejorar sus características operativas cuando se emplean de forma acumulativa y si se tiene en cuenta su poca utilidad en pacientes ancianos, pacientes de sexo masculino. También mejoran sus características si se observan con rigor las denominadas «señales de alarma».

En 1992 y 1999 se reunió en Roma un grupo de trabajo internacional que publicó los resultados de un consenso de definiciones de los trastornos funcionales del aparato digestivo, dentro de los cuales está el SII, y que definió para éste los criterios de Roma I y Roma II ${ }^{3,58,66}$ (tabla V). Estos criterios, los más utilizados hasta el momento, al menos en investigación, no han sido validados en su constructo interno y externo en nuestros países, pero son aparentemente más sensibles y específicos que los de Manning et $\mathrm{al}^{2,56}$ y centran el proceso diagnóstico en la presencia, durante al menos 12 semanas, consecutivas o no en los últimos 12 meses, de dolor abdominal asociado a 2 de 3 posibles cambios en el hábito intestinal (mejoría con la defecación, inicio asociado a cambios en la frecuencia o en la consistencia). Para aumentar su valor predictivo positivo, sensibilidad y especificidad, se postulan 5 síntomas adicionales ${ }^{58}$ (tabla V).

\section{Evaluación inicial}

El proceso diagnóstico se inicia con la comprobación de la presencia de los criterios diagnósticos (Maning et al o Roma II) en un paciente sin signos de enfermedad orgánica evidente ${ }^{67}$. Para evitar el uso excesivo de recursos en una entidad muy frecuente, se recomienda una evaluación global, agregando los siguientes exámenes de laboratorio $^{68}$ : a) hemograma completo con velocidad de sedimentación globular; $b$ ) determinación de la hormona tirostimulante; c) muestras fecales para detección de parásitos, y d) sigmoidoscopia flexible, o enema de bario o colonoscopia si el paciente es mayor de 50 años.

Sin embargo, el American College of Gastroenterology $y^{67}$ no comparte este abordaje y propone que sólo se aplique a pacientes con «señales de alarma», que pueden ser las referidas en la tabla VI. La presencia de cualquiera de estas señales de alarma casi automáticamente excluye la posibilidad diagnóstica del SII.

La forma y el tiempo de indicar los estudios diagnósticos que excluyan una enfermedad orgánica dependen casi siempre del síntoma predominante. El uso juicioso de los criterios sumado a los estudios iniciales permite establecer un diagnóstico de inclusión en el $95 \%$ de los pacientes con SII; es decir, sólo se diagnostica incorrectamente de SII a un 5\% de los pacientes con enfermedad orgáni$\mathrm{ca}^{67,68}$, entre los que se incluyen aquellos que tienen enfermedad psiquiátrica predominante. La valoración periódica del paciente permite disminuir aún más los falsos positivos al utilizar la combinación de criterios y evaluación paraclínica inicial ya descrita.

\section{Diagnóstico diferencial}

Algunos autores ${ }^{69}$ proponen centrar en 5 grandes puntos el diagnóstico diferencial del SII (tabla VII), el cual habitualmente puede realizarse con las pruebas paraclínicas usadas en la evaluación inicial o ante la presencia de señales de alarma.

En resumen, el uso de los criterios de Roma II y la ausencia de signos de alarma permiten llegar al diagnóstico de inclusión del SII. Para el manejo se debe tener en cuenta el síntoma predominante (estreñimiento, diarrea o alter- 
TABLA VII. Diagnóstico diferencial del síndrome del intestino irritable

Malabsorción

Intolerancia a alimentos

Infecciones entéricas

Enfermedad inflamatoria intestinal

Trastornos psiquiátricos

nancia de ambos), según criterios de Roma II. La epidemiología local obligará a descartar algunas entidades como son las parasitosis o la enfermedad celíaca. Las personas mayores de 50 años con signos de alarma deben someterse al esquema de diagnóstico diferencial.

\section{GENERALIDADES SOBRE EL TRATAMIENTO}

Una vez que se ha diagnosticado el SII positivamente y se han excluido otras patologías, de la forma más directa y económica posible, debe recalcarse al paciente la importancia y el significado de este diagnóstico. El médico debe ser asertivo en su diagnóstico y tratar de establecer una óptima relación con el paciente. Puede ser de especial relevancia asegurar al paciente que el SII es un proceso benigno y que no hay evidencias de cáncer, así como explicarle que no es necesario indicar cirugía ni realizar otros exámenes más invasivos y costosos, e informarle del curso crónico recurrente que puede tener el SII y de los factores precipitantes de las crisis, como son las transgresiones alimentarias y los períodos de estrés.

De forma muy general, según su gravedad, los casos de SII se pueden categorizar en leves, moderados y graves. Las medidas terapéuticas utilizadas deben estar en relación con la gravedad del cuadro y estar orientadas a mejorar la calidad de vida del paciente, siempre considerando el gasto que puede ocasionar ${ }^{70}$.

Es recomendable explicar con vocabulario simple la anatomía abdominal, los conceptos de hipersensibilidad visceral, alteraciones motoras y secretoras intestinales que ocurren en el SII, la importancia del estrés y de los cambios dietéticos, y la posible relación con las infecciones intestinales. El nivel de información que se proporcione en esta fase debe estar determinado por el grado de educación y receptividad del paciente y de sus expectativas al consultar. En las formas leves es posible que una adecuada explicación de los puntos señalados, acompañada de tratamiento sintomático, sea suficiente. Si no, el buen juicio clínico indicará las distintas formas de tratamiento que analizaremos a continuación.

Para recomendar un tratamiento, éste debe haberse evaluado con criterios rigurosos, en estudios aleatorizados y de doble ciego (el médico tratante y el paciente) con una duración aceptable y un número adecuado de pacientes, y en los resultados debe analizarse a todos los pacientes a los que se ha intentado tratar (véanse los grados de evidencia y grado de recomendación en la tabla I).

En resumen, el tratamiento del SII debe iniciarse estableciendo una buena relación médico-paciente, debe ser integral e incluir recomendaciones para una posible modificación del estilo de vida. El tratamiento farmacológico debe estar enfocado según los síntomas predominantes, el motivo de consulta y el grado de afectación de la calidad de vida del paciente, y se elegirá en la medida de lo posible el que tenga una mejor relación coste-beneficio.

\section{Dieta}

Si bien no hay evidencias que apoyen científicamente los cambios de dieta, se ha observado que en algunos pacientes mejoran los síntomas con modificaciones dietéticas. En la población adulta latinoamericana el déficit de disacaridasas (lactasa y otras) es relativamente alto, por lo que hay que interrogar al paciente sobre la intolerancia a la leche y sus derivados. Si esta relación no es muy evidente, se puede recomendar la supresión de lácteos, lo que en algunos casos da lugar a la mejoría total de los síntomas. Obviamente, en los casos con intolerancia a la lactosa debe reconsiderarse el diagnóstico de SII. La evaluación de la propuesta dietética debe tener en cuenta el efecto placebo.

\section{TRATAMIENTOS FARMACOLÓGICOS}

\section{Efectividad de los antiespasmódicos y relajantes musculares}

El empleo de los antiespasmódicos se basa en la premisa de que al relajar el músculo liso intestinal disminuyen el dolor abdominal y los síntomas asociados al espasmo, y por esta razón han sido y continúan siendo ampliamente utilizados, ya sea solos o asociados a otros fármacos como los analgésicos o sedantes, en el tratamiento del dolor cólico abdominal o de la diarrea. El dolor o malestar abdominal es uno de los síntomas cardinales del SII y en la fisiopatología clásica se achacaba este síntoma a trastornos motores o espasmos; de ahí la denominación de «colon espasmódico».

\section{Clases de antiespasmódicos}

Antimuscarínicos no específicos. Bloquean de forma competitiva los receptores muscarínicos impidiendo la acción de la acetilcolina sobre el músculo liso y las secreciones exocrinas. En los aparatos gastrointestinal y genitourinario, determinan una reducción del tono muscular y de la amplitud y frecuencia de las contracciones. Entre estos fármacos se incluyen la atropina, hioscina, hiosciamina, diciclomina homatropina y el propinoxato.

Pueden producir efectos adversos que incluyen taquicardia, sedación, confusión, visión borrosa, sequedad de boca, intolerancia al calor y retención urinaria particularmente en ancianos.

Antimuscarínicos específicos. Los antimuscarínicos intestinales específicos son los compuestos de amonio cuaternario que se absorben mal a través de la mucosa; de esta forma actúan en el músculo liso intestinal con menores efectos adversos. Entre estos agentes figuran el bromido 
cimetropio, bromido de pinaverio y bromido octilonio. Los 2 últimos tienen una actividad significativa como bloqueadores de los canales del calcio.

Bloqueadores del calcio. El bromuro de pinaverio, además de un efecto antimuscarínico, presenta un efecto inhibidor de los canales del calcio. La selectividad intestinal se atribuye a su baja absorción y rápido metabolismo hepático. Este bromuro reduce la motilidad colónica posprandial o inducida por neostigmina e incrementa el tiempo de tránsito intestinal sin afectar el carácter de las deposiciones $^{71}$.

El bromuro de octilonio interfiere con la disponibilidad de los iones de calcio, reduce la motilidad sigmoidea posprandial y mejora tanto el dolor como la sensación de distensión. Tanto el bromuro de octilonio como el de pinaverio carecen de acciones antimuscarínicas sistémicas.

Opiáceos. La trimebutina se une a los receptores $\mu, \lambda, \delta$ y modula la actividad colinérgica y adrenérgica; se postula que actúa directamente en el músculo intestinal liso. Puede prevenir los cambios motores en el complejo migratorio motor posprandial o el inducido por estrés, reduce la actividad colónica en los pacientes con SII y acelera el tiempo de tránsito intestinal en pacientes con estreñimiento ${ }^{72}$.

Antiespasmódicos sin acción anticolinérgica. La mebeverina actúa como antiespasmódico mediante el bloqueo de los canales de sodio que permiten la entrada de calcio, lo que finalmente conduce a la contracción muscular. Secundariamente reduce la salida de potasio de la célula, lo que evita la hipotonía. No posee efectos adversos antimuscarínicos.

\section{Revisión de la evidencia de la efectividad de los antiespasmódicos en el tratamiento del síndrome del intestino irritable}

En un estudio doble ciego y controlado con placebo realizado en México $^{73}$, se administró pinaverio a 40 pacientes con SII (20 en cada grupo), durante 3 semanas. Se comprobaron una disminución significativa de la duración del dolor y una mejoría de la distensión abdominal en el grupo que recibió pinaverio al compararlo con el que recibió placebo.

En un metaanálisis ${ }^{74}$ se revisó la efectividad de 8 medicamentos: cimetropio, diciclomina, pinaverio, mebeverina, hioscina, octilonio, extracto de menta y trimebutina. Sólo 5 fármacos (cimetropio, mebeverina, octilonio, pinaverio y trimebutina) mostraron eficacia clínica, sin efectos adversos. Independientemente del tipo de miorrelajante investigado, éstos son más efectivos que el placebo para lograr una mejoría global del paciente. Los miorrelajantes son también útiles en el tratamiento del dolor; sin embargo, no fueron significativamente eficaces en el tratamiento de la distensión abdominal. La diciclomina y la hioscina no fueron significativamente eficaces.

Otro metaanálisis ${ }^{75}$ revisó la efectividad de 6 fármacos sin efectos secundarios significativos (cimetropio, hiosci- na, meveberina, octilonio, pinaverio y trimebutina). Se confirmó la utilidad clínica de los miorrelajantes en el SII teniendo en cuenta los parámetros de mejoría global del dolor y en menor grado de la distensión. No hubo diferencia significativa en el análisis de las alteraciones del tránsito intestinal (estreñimiento o diarrea).

Por último, otro análisis más restringido ${ }^{76}$, que se limitó únicamente a estudios publicados en inglés, concluyó que los miorrelajantes son beneficiosos cuando el dolor es el síntoma predominante; sin embargo, alguno de ellos, como la diciclomina, presenta un porcentaje inaceptable de efectos secundarios de tipo anticolinérgico. Éstos no se observaron con el pinaverio o el cimetropio.

Uno de los problemas respecto al diseño de los estudios aleatorizados para el tratamiento del SII es la respuesta al placebo, que es extremadamente variable y oscila entre un $40 \mathrm{y}$ un $70 \%$.

Puesto que el SII es una enfermedad crónica con períodos impredecibles de exacerbación y remisión, los trabajos limitados a pocas semanas son de escasa relevancia para establecer que un tratamiento es efectivo en un plazo prolongado. Si se analizan los trabajos incluidos en estos metaanálisis siguiendo los criterios de diseño metodológico recomendado para los trastornos funcionales ${ }^{1}$, la mayoría de los estudios incluidos presenta errores metodológicos que limitan sus resultados ${ }^{77}$. Así, por ejemplo, en una revisión de trabajos aleatorizados ${ }^{78}$ que incluyó estudios en varios idiomas, sólo 2 trabajos con fármacos antiespasmódicos, ambos con cimetropio, reúnen criterios de calidad. En ambos se observó una mejoría global significativa.

En otra revisión clínica publicada ${ }^{79}$ se observó que, si bien algunos antiespasmódicos fueron superiores al placebo en términos de mejoría global de los síntomas y en el alivio del dolor, la mayoría de los trabajos revisados tuvieron una duración menor de 8 semanas, por lo que eran cuestionables metodológicamente. Además podría existir un «sesgo de publicación». Se concluyó que el beneficio de los antiespasmódicos es probablemente, cuando mucho, modesto. A pesar de esto, en la revisión se afirma que en la práctica clínica podrían ser útiles para los episodios predecibles e intermitentes de dolor posprandial, pero que frecuentemente fallan a la hora de resolver síntomas más complejos.

En las revisiones sistemáticas más recientes ${ }^{67}$ se concluye que los datos sobre la efectividad de los antiespasmódicos disponibles en EE.UU., esto es, diciclomina e hiosciamina, son insuficientes. Por lo tanto, del resultado de la revisión de la evidencia sobre la efectividad de los antiespasmódicos en el tratamiento del SII se deriva que los antiespasmódicos anticolinérgicos, dados sus efectos adversos y escaso beneficio, no son fármacos adecuados. No hay suficientes trabajos con métodos adecuados que avalen el uso de los antiespasmódicos sin efecto anticolinérgico, sobre todo si el objetivo es la mejoría global de los síntomas del SII, aunque podrían ser útiles en el tratamiento de síntomas específicos, sobre todo el dolor. Por carecer de efectos adversos, todavía desempeñan un papel en el tratamiento integral del SII (recomendación de grado B). 


\section{Efectividad de las fibras y agentes que aumentan el volumen del contenido intestinal}

El uso de dietas ricas en fibras para tratar una variedad de síntomas gastrointestinales y algunas observaciones anecdóticas han fomentado la creencia de que los trastornos de la motilidad intestinal en pacientes con SII pueden ser reversibles agregando fibras a la dieta ${ }^{80}$. En estudios sobre dietas, en los que se ha administrado, por ejemplo, trigo como fuente de fibra, se ha observado que el tránsito intestinal se aceleró en voluntarios sanos, presumiblemente porque se habría incrementado la cantidad de líquido intraintestinal ${ }^{81,82}$. Sin embargo, si bien la dieta con fibra mejora el tránsito intestinal y el contenido líquido de las deposiciones, no tiene efecto beneficioso sobre otros síntomas que acompañan al SII, como es la distensión abdominal. A este respecto, un estudio aleatorizado, doble ciego y con placebo demostró que el trigo agrava la sensación de distensión abdominal y la flatulencia ${ }^{83}$.

Se han revisado publicaciones en las que se analizó el efecto de las siguientes fibras en el tratamiento del SII: psilio $^{84,85}$, trigo en fibra ${ }^{86,87}$, galletas de trigo (de 10 a 15,6 g/día $\left.{ }^{88-90}\right)$, fibra concentrada (4,1 g/día $\left.{ }^{91}\right)$, fibra de maíz (100 $\mathrm{mg} /$ día $\left.^{92}\right)$, calcium polycarbophil (6 g/día $\left.{ }^{93}\right)$ e ispaghl ${ }^{94,95}$. Sólo 4 de los 12 estudios realizados con fibra o agentes que aumentan el volumen intestinal demostraron que éstos carecían de un beneficio consistente en el SII. En todos los estudios la mejoría estadística comunicada se refería a una serie de síntomas no específicos como, por ejemplo, frecuencia y sensación de evacuación satisfactoria, pero es importante hacer notar que no se encontró mejoría en síntomas más específicos como el dolor y la distensión abdominal.

Hasta el momento, la fibra y los agentes que aumentan el volumen intestinal no han demostrado tener un beneficio terapéutico, incluso pueden aumentar el dolor, la distensión y el meteorismo, y su indicación sería sólo para ayudar en mejorar el estreñimiento (recomendación de grado B).

\section{Efectividad de los antidiarreicos}

El SII con predominio de diarrea se caracteriza por un tránsito acelerado del intestino delgado y colon proximal, lo que se traduce en deposiciones líquidas, urgencia defecatoria y secreción rectal. Para el tratamiento de estos síntomas existen fármacos que disminuyen la motilidad intestinal y aumentan la reabsorción de agua y electrólitos. En general, se recomienda que deben usarse estratégicamente para episodios de diarrea que interfieran con el trabajo o la vida social y que no hayan respondido a otras medidas terapéuticas previas. No producen alivio del malestar ni del dolor abdominal. Si hacemos una revisión crítica de las publicaciones sobre este tema, no encontraremos trabajos de nivel I que apoyen el uso de los antidiarreicos en el tratamiento del SII.

\section{Loperamida}

Es el fármaco de primera elección para el tratamiento del SII con predominio de diarrea. La loperamida es un opiá- ceo sintético derivado de la butiramida que disminuye el tránsito intestinal actuando sobre la musculatura longitudinal y circular del intestino; además, aumenta la absorción de agua e iones, mejora la consistencia de las heces e incrementa el tono del esfínter anal, lo que da lugar a una mejoría de la diarrea ${ }^{96}$. El efecto sobre el tono en reposo del esfínter anal ayuda a reducir la secreción rectal nocturna. El mecanismo principal de continencia fecal está dado por el esfínter anal interno ${ }^{97,98}$. La loperamida tiene la ventaja sobre otros opiáceos como el difenoxilato o la codeína de que a dosis estándar no tiene efectos opiáceos, ya que atraviesa mínimamente la barrera hematoencefálica. A pesar de que un reciente estudio mostró que la loperamida disminuyó el dolor además de las deposiciones líquidas, la mayoría de los estudios controlados y aleatorizados sólo han demostrado mejoría en la frecuencia de las deposiciones y no en el dolor $^{98}$ (recomendación de grado $B$ ).

\section{Difenoxilato}

A dosis de 2,5 mg, de 3 a 4 veces al día, reduce la frecuencia y urgencia de las deposiciones. A dosis más bajas disminuye la acción opiácea sobre el SNC. El problema radica en que, al contener atropina, ésta puede tener efectos adversos, especialmente en los ancianos que presentan disfunción vesical, glaucoma o taquicardia, por lo que su uso se limita a los pacientes que no toleren la loperamida. No se ha evaluado en el tratamiento del SII.

\section{Colestiramina}

Considerada como la tercera línea de tratamiento, la colestiramina, un secuestrador de sales biliares, se usa para un subgrupo de pacientes que presentan malabsorción de sales biliares o están colecistectomizados y cursan con diarrea. El principio se basa en la documentación de malabsorción de ácidos biliares en algunos pacientes con diarrea dolorosa semejante al SII con diarrea. Este subgrupo se diagnostica ya sea por ensayo terapéutico o medición sérica de la 7-alfa-hidroxicolesten-3-uno; este último examen sólo se realiza en centros especializados (recomendación de grado $C$ ).

\section{Otros agentes antidiarreicos}

Son varios los fármacos que se han probado pero que se han dejado de lado porque no muestran beneficios mayores que los antidiarreicos clásicos o tienen muchos efectos secundarios. Entre ellos se encuentra la lidamidina, un agonista adrenérgico $\alpha_{2}$ que estimula el sistema adenilciclasa, regula el flujo de calcio y disminuye la secreción de agua y electrólitos ${ }^{99,100}$. Se suspendió su uso por presentar efecto cardiológico secundario importante. Posteriormente se probó con la clonidina, otro agonista adrenérgico central $\alpha_{2}$ cuyo efecto es relajar el colon izquierdo, reducir el umbral del dolor e incrementar la absorción de líquidos; sin embargo, el problema principal de este fármaco es que produce hipotensión postural importante asociada a fatiga ${ }^{101}$. 


\section{Efectividad de los antidepresivos tricíclicos}

La casi totalidad de los estudios que han empleado antidepresivos en el SII lo han hecho con antidepresivos tricíclicos (ATC), y prácticamente no se han publicado estudios (sólo casos aislados en forma de resumen) sobre los inhibidores selectivos de recaptación de serotonina (fluoxetina y otros).

Hay un pequeño grupo de pacientes que presentan síntomas gastrointestinales graves y muchos de los cuales tienen trastornos psicológicos (depresión, ansiedad, crisis de pánico), y es en este grupo en el que los antidepresivos pueden desempeñar un papel importante. Estos fármacos pueden reservarse para pacientes refractarios a otros tratamientos cuyo síntoma primordial sea el dolor abdominal frecuente y grave, y en algunos casos con diarrea de difícil manejo ${ }^{102}$.

Los antidepresivos tienen propiedades neuromoduladoras y analgésicas (tanto somáticas como viscerales), y éstas son independientes de su efecto psicotrópico o antidepresivo. Los mecanismos que se postulan en la modulación del dolor en el SII con ATC es la facilitación de descarga de endorfinas endógenas, el bloqueo de la recaptación de noradrenalina, con lo que se incrementan las vías inhibitorias dolorosas descendentes, y el bloqueo de la recaptación del neuromodulador del dolor, la serotonina.

Los ATC, gracias a sus propiedades anticolinérgicas, también enlentecen el tiempo de tránsito intestinal y pueden ser útiles en el SII con predominio de diarrea; por el contrario, pueden agravar a pacientes con estreñimiento.

Dentro de los ATC existen derivados de aminas secundarias, tales como la nortriptilina y desipramina, y derivados de aminas terciarias, como la amitriptilina e imipramina. Las aminas secundarias tienen menos efectos colaterales como sedación, efectos antihistamínicos y anticolinérgicos, ortostatismo, aumento de peso, etc.

Las dosis de ATC están en el rango de 25-100 mg/día, comenzando con 10-25 mg/día para luego ir titulando a cada paciente en función de los efectos secundarios y la respuesta. Deben administrarse siempre por la noche. El inicio de su acción analgésica puede ser muy temprano (del primer al séptimo días), pero otros pacientes requieren de 3 a 4 semanas de tratamiento antes de ver su efecto total, de ahí la importancia de no considerar que el tratamiento ha fracasado antes de los plazos mencionados. Una vez lograda la respuesta, el tratamiento debe mantenerse durante un período de 6-12 meses para luego suspenderlo lentamente.

El uso óptimo de antidepresivos está en el contexto de una estrecha relación médico-paciente, y estos fármacos deben verse como un complemento a un plan de tratamiento global. Deben explicarse claramente al paciente la razón de su uso, los posibles efectos secundarios, que habitualmente son muy tempranos (de uno a 2 días) y que no siempre obligan a su suspensión, ya que muchos de ellos ceden a los pocos días, y los beneficios esperados del fármaco prescrito. La respuesta al tratamiento antidepresivo es altamente específica del paciente, de modo que no es posible predecir quién responderá a él o quién experimentará efectos adversos.
Klein ${ }^{103}$ revisó la bibliografía en inglés hasta 1986 sobre el uso de los diversos ATC en el SII, y comentó que todos los trabajos hasta esa fecha adolecían de serios fallos metodológicos, entre los que figuraban la falta de claridad de los criterios de inclusión (antes de que se establecieran los criterios de Roma), el reducido tamaño de las muestras (considerando el gran efecto placebo en esta afección), muchos abandonos (debido a importantes efectos secundarios o falta de respuesta), períodos de estudio cortos (teniendo en cuenta la latencia de respuesta con estos fármacos), inadecuado diseño de estudio o de uso de análisis estadísticos. Hay que considerar que el efecto placebo puede llegar a un 40-70\%. Según Klein ${ }^{103}$, ni un solo estudio ofrece evidencias convincentes de que algún tipo de tratamiento sea efectivo en el complejo sintomático del SII, y reconoce que los ATC podrían ayudar a tratar síntomas aislados tales como dolor abdominal o diarrea.

En otro estudio se administró tratamiento antidepresivo a 138 pacientes con SII en una experiencia clínica de 5 años. Se usaron hasta 5 fármacos diferentes de forma consecutiva si no había respuesta, y se observó que había mejoría y remisión completa de los síntomas intestinales en un 89 y un $61 \%$, respectivamente, durante el tratamiento ${ }^{104}$. Cuando el dolor abdominal era el síntoma predominante, hubo mayor remisión de síntomas (un $81 \%$ de los pacientes con predominio de dolor abdominal mejoró, en comparación con sólo el $60 \%$ en que predominaba la diarrea). Sin embargo, dado su diseño, este estudio retrospectivo no fue capaz de determinar la eficacia de los antidepresivos en el SII.

Un metaanálisis que incluyó 12 trabajos que habían empleado antidepresivos en el SII, controlados con placebo ${ }^{105}$, concluyó que los ATC se asociaron con mejoría de los síntomas. Se usaron la amitriptilina, clomipramina, desipramina, doxepina y trimipramina. La odds ratio final para la mejoría con ATC fue de 4,2. En promedio fue preciso tratar a 3,2 pacientes para mejorar los síntomas de uno.

Por otra parte, una revisión sistemática de 6 trabajos controlados y aleatorizados sobre el uso de ATC (desipramina, amitriptilina, trimipramina y doxepina) en el SII concluyó que estos fármacos no eran más efectivos que el placebo a la hora de mejorar los síntomas globales del SII. Sin embargo, la evidencia limitada apoyó un posible beneficio de los ATC en la mejoría del dolor abdominal. En estos trabajos las dosis empleadas fueron adecuadas para el tratamiento del dolor crónico, pero el diseño fue subóptimo (de acuerdo con las recomendaciones de Roma), el tamaño de la muestra fue pequeño (menos de 31 pacientes en cada grupo del estudio) y la duración del estudio fue corta (4-8 semanas) ${ }^{106}$.

Una revisión sistemática ${ }^{107}$ de todos los trabajos controlados y aleatorizados de farmacoterapia en el SII publicados de 1966 a 1999 logró identificar 28 trabajos, y sólo uno de ellos demostró beneficio con los ATC y se consideró aceptable. Una revisión reciente ${ }^{67}$ concluye respecto al uso de los ATC en el SII que estos fármacos no son más efectivos que el placebo en la mejora de los síntomas globales de la enfermedad, pero mejoran el dolor abdominal de algunos de estos pacientes (tabla VIII). 
TABLA VIII. Características farmacológicas de los antidepresivos tricíclicos empleados en el síndrome del intestino irritable

\begin{tabular}{|l|c|c|c|c|}
\hline Fármaco & Anticolinérgico & Serotonínico & Antihistamínico & Dosis (mg) \\
\hline Amitriptilina & \multicolumn{2}{|c|}{} & +++ & $10-50$ \\
Desipramina & +++ & +++ & + & $25-125$ \\
Doxepina & + & +++ & +++ & $50-75$ \\
Maprotilina & ++ & +++ & ++++ & $50-100$ \\
Nortriptilina & + & Sin & ++ & $25-125$ \\
\hline
\end{tabular}

No se ha podido localizar trabajos latinoamericanos que hayan estudiado el efecto de los ATC en el SII.

\section{Efectividad de los antagonistas $5-\mathrm{HT}_{3}$ de la serotonina (alosetrón, granisetrón, ondansetrón y cilansetrón)}

Los receptores $5-\mathrm{HT}_{3}$ son canales iónicos unidos a ligandinas que facilitan la acción despolarizante de la serotonina, lo que da lugar a la liberación del neurotransmisor. Son similares en su estructura a los receptores nicotínicos de la acetilcolina. Se localizan tanto en el SNC, en el sistema límbico, corteza y centro del vómito, como en el tracto gastrointestinal, donde se ubican en las fibras sensoriales aferentes y en las neuronas entéricas postsinápticas de los plexos submucoso y mientérico. Además, se encuentran en la raíz dorsal de las neuronas ganglionares y transmiten sensibilidad del intestino a la médula espinal $^{108}$.

\section{Antagonistas del receptor 5-HT3: ondansetrón, granisetrón, tropisetrón, alosetrón y cilansetrón}

Son los más conocidos y estudiados. Los antagonistas del receptor 5- $\mathrm{HT}_{3}$ actúan en aferentes vagales y en receptores centrales en la zona quimiorreceptora y centro del vómito, lo que da lugar al control de los vómitos asociados con la radioterapia y quimioterapia ${ }^{108,109}$. El antagonismo de los receptores 5- $\mathrm{HT}_{3}$ en la zona intestinal reduce el dolor visceral, retrasa el tránsito colónico y mejora la absorción en el intestino delgado. Al actuar en la motilidad intestinal los antagonistas de los receptores $5-\mathrm{HT}_{3}$ disminuyen las contracciones; en el aparato sensorial reducen la sensación visceral y tienen el potencial de restaurar la sensibilidad y la función motora en el SII ${ }^{108}$.

$\mathrm{El}$ ondansetrón fue el primer antagonista $5-\mathrm{HT}_{3}$ que se evaluó en el SII con predominio de diarrea. Disminuyó el tránsito colónico, pero no modificó el dolor abdominal ${ }^{109}$. El granisetrón aumentó el umbral del volumen rectal, pero no tuvo acción sobre el dolor abdominal. El tropisetrón no se ha estudiado en el tratamiento del SII. El ondansetrón, granisetrón y tropisetrón sólo están indicados como potentes antieméticos en emesis asociada a quimioterapia o radioterapia ${ }^{109}$.

El alosetrón y últimamente el cilansetrón se han investigado en el SII con predominio de diarrea. Tienen efectos positivos en el alivio del dolor abdominal y disminuyen tanto el tránsito colónico como la secreción de agua y cloruros. El alosetrón es un antagonista selectivo de los receptores $5-\mathrm{HT}_{3}$, que efectivamente alivia el dolor abdo- minal, normaliza el número de evacuaciones intestinales y la urgencia en mujeres con SII con predominio de diarrea. Se supone que el mecanismo y los sitios de acción del alosetrón parten de su interferencia con la activación de los receptores periféricos $5-\mathrm{HT}_{3}$. Sin embargo, se ha comprobado que la reducción de los síntomas se correlaciona con menor actividad en las redes nerviosas centrales que median la expresión emocional ${ }^{110}$.

En estudios controlados y doble ciego, el alosetrón a dosis de $1 \mathrm{mg} 2$ veces al día ha sido más efectivo que el placebo en el alivio del dolor, de la distensión abdominal, de la frecuencia, consistencia y urgencia en las evacuaciones, con mejoría en la calidad de vida de las pacientes tratadas. En estudios de 12 semanas, la mejoría del dolor y del número de evacuaciones se manifiesta entre la primera y cuarta semanas de tratamiento, y los síntomas reaparecen una semana después de interrumpido el tratamiento con una frecuencia similar a la del grupo placebo. Todos los estudios controlados han demostrado resultados superiores en el sexo femenino y sólo se ha aprobado su uso en mujeres con SII con predominio de diarrea ${ }^{110}$.

El efecto adverso más común observado con el alosetrón es el estreñimiento (28\%), en comparación con placebo (5\%). El estreñimiento suele ser de leve a moderado y sólo el $10 \%$ de los pacientes incluidos en estudios de fase III se retiraron por ello ${ }^{110}$. Una complicación grave sin relación precisa con el alosetrón es la colitis isquémica aguda. Su frecuencia se estima entre un 0,1 y un $1 \%$ de los pacientes. No se han identificado los factores de riesgo para desarrollar esta complicación. Los casos publicados se resolvieron, aunque se produjeron 5 muertes en pacientes por colitis isquémica que estaban tomando alosetrón y otros medicamentos, lo que motivó la retirada del mercado de este fármaco en noviembre del año $2000^{111}$. En junio de 2002, la Food and Drugs Administration aprobó de nuevo el uso restringido de alosetrón para mujeres con diarrea grave asociada a SII en las que han fallado los tratamientos convencionales. Hasta este momento, los médicos que lo utilicen deben completar cuestionarios educativos y recetarios especiales, y se debe obtener el consentimiento firmado de la paciente ${ }^{111}$.

El cilansetrón, otro antagonista del receptor $5-\mathrm{HT}_{3}$, se está evaluando en estudios de fase III para varones y mujeres con SII con predominio de diarrea. Ha demostrado poseer una eficacia similar al alosetrón en 2 estudios de fase II y fue efectivo en el sexo masculino, probablemente porque se estudió en un mayor número de varones ${ }^{112}$. En conclusión, los antagonistas del receptor 5- $\mathrm{HT}_{3}$ de la serotonina mejoran los síntomas, especialmente en mujeres con SII con predominio de diarrea. Disminuyen el do- 
TABLA IX. Características de los ensayos clínicos aleatorizados en fase III sobre el tegaserod frente a placebo en el síndrome del intestino irritable (SII)

\begin{tabular}{|c|c|c|c|c|}
\hline Autor & $\begin{array}{l}\text { Dosis de tegaserod } \\
\text { (mg/día) }\end{array}$ & Pacientes (\% mujeres) & Parámetros evaluados & Respuesta \\
\hline $\begin{array}{l}\text { Muller-Lissner et al }{ }^{118} \\
\text { Estudio }{ }^{\circ}{ }^{\circ} \mathrm{B} 301\end{array}$ & 4 o 12 & $881(83)$ & $\begin{array}{l}\text { Mejoría global del SII, síntomas individuales, } \\
\text { efectos adversos }\end{array}$ & $\begin{array}{l}\text { Tegaserod: } 38,4 \% \text {; placebo: } \\
30,2 \%\end{array}$ \\
\hline $\begin{array}{l}\text { Lefkowitz et al }{ }^{119} \\
\text { Estudio } n .^{\circ} \text { B351 }\end{array}$ & 4 o 12 & $799(87)$ & Dolor y malestar abdominales & $\begin{array}{l}\text { Tegaserod: } 46 \% \text {; placebo: } \\
33 \%\end{array}$ \\
\hline $\begin{array}{l}\text { Estudio n. }{ }^{\circ} 307^{123} \\
\text { Estudio n. }{ }^{\circ} \text { B307 }\end{array}$ & 4 & $841(84)$ & $\begin{array}{l}\text { Mejoría global del SII, síntomas individuales, } \\
\text { efectos adversos }\end{array}$ & $\begin{array}{l}\text { Tegaserod: } 42,2 \% \text {; placebo: } \\
37 \%\end{array}$ \\
\hline $\begin{array}{l}\text { Novicki et al }{ }^{120} \\
\quad \text { Estudio n. }{ }^{\circ} \text { B358 }\end{array}$ & 12 & $1.519(100)$ & $\begin{array}{l}\text { Mejoría global del SII, síntomas individuales, } \\
\text { efectos adversos }\end{array}$ & $\begin{array}{l}\text { Tegaserod: } 43,5 \% \text {; placebo: } \\
\quad 38,8 \%\end{array}$ \\
\hline $\begin{array}{l}\text { Kellow et al }{ }^{121} \\
\quad \text { Estudio n. }{ }^{\circ} \text { ASG (ZAP) }\end{array}$ & 12 & $520(88)$ & $\begin{array}{l}\text { Mejoría global del SII, síntomas individuales, } \\
\text { efectos adversos }\end{array}$ & $\begin{array}{l}\text { Tegaserod: } 47 \% \text {; placebo: } \\
28 \%\end{array}$ \\
\hline $\begin{array}{l}\text { Nyhlin et } \text { al }^{122} \\
\text { Estudio n. } \\
\text { AFI01 (TENOR) }\end{array}$ & 12 & $647(86)$ & $\begin{array}{l}\text { Mejoría global del SII, síntomas individuales, } \\
\text { efectos adversos }\end{array}$ & Datos no disponibles \\
\hline
\end{tabular}

lor abdominal, el número de evacuaciones y la urgencia. El alosetrón y últimamente el cilansetrón son los que actúan en la región intestinal. En el momento actual, su uso puede recomendarse con mucha cautela cuando las pacientes que presentan SII con predominio de diarrea no responden a medidas convencionales como son la dieta, anticolinérgicos, loperamida, etc. Al usarlos se deben vigilar estrechamente las reacciones secundarias, como el estreñimiento grave y la colitis isquémica, y hay que interrumpir el tratamiento si la paciente presenta hemorragia intestinal (recomendación de grado A). No se comercializa en Latinoamérica.

\section{Tratamiento con los agonistas $5-\mathrm{HT}_{4}$ de la serotonina (tegaserod)}

El tegaserod es un agonista parcial selectivo de los receptores tipo 4 de la serotonina $\left(5-\mathrm{HT}_{4}\right)$ en el tubo digestivo $^{113,114}$. Tiene efectos procinéticos en el estómago, intestino delgado y colon, y acciones antinociceptivas viscerales ${ }^{115}$. En pacientes con SII, el tegaserod acelera el tránsito en el intestino delgado y tiende a disminuir el tiempo de tránsito colónico ${ }^{116}$.

La eficacia del tegaserod frente a placebo se ha evaluado en 6 ensayos clínicos aleatorizados y doble ciego de fase III en más de 5.000 pacientes con SII con predominio de estreñimiento (tabla IX) ${ }^{118-123}$. En la mayoría de los estudios se incluyó predominantemente a pacientes de sexo femenino $(>80 \%)$, de raza caucásica $(>80 \%)$ y con una edad media de 45 años $^{118-120,122}$. En un estudio recientemente publicado se evaluó a 520 pacientes con SII sin diarrea de las regiones de Asia y del Pacífico (Australia, China, Indonesia, Malasia, Corea, Filipinas y Tailandia), de los cuales el $88 \%$ eran mujeres y el $84 \%$ de raza no caucásica $^{121}$. En estos estudios se demostró que el tegaserod, a dosis de $6 \mathrm{mg} 2$ veces al día durante 12 semanas, mejoraba significativamente los síntomas globales del SII comparado con el placebo. El beneficio terapéutico sobre el placebo fue del 5 al 19\% en los diferentes estudios. En el análisis por subgrupo de síntomas, los pacientes tratados con tegaserod tuvieron una mejoría significativa en la distensión, en el dolor o malestar abdominales y en los hábitos intestinales (mayor frecuencia y mejor consistencia de las evacuaciones) comparados con los pacientes que recibieron placebo ${ }^{118-123}$. La magnitud de la respuesta varió entre los estudios debido al uso de diferentes escalas para evaluar los síntomas individuales del SII. Existe una cierta experiencia en Latinoamérica ${ }^{124}$.

La respuesta al tegaserod fue rápida, ya que se observó una mejoría sintomática en la mayoría de los pacientes desde la primera semana, y el efecto se mantuvo durante las 12 semanas de tratamiento. Tras suspender el tegaserod se produjo una pérdida significativa del efecto terapéutico después de la primera semana sin recibir el fármaco, pero no se demostró un efecto de rebote de los síntomas al interrumpir el tratamiento ${ }^{123}$.

El tegaserod fue más efectivo en mujeres y, aunque hubo cierta mejoría en los varones, ésta no fue significativamente superior a la del placebo. El reducido tamaño de las muestras de varones en los estudios de fase III y un posible error tipo II pudieran explicar la falta de significación estadística en la eficacia terapéutica del tegaserod en los pacientes masculinos ${ }^{118-123}$.

No se han comunicado efectos adversos graves con el tegaserod. La diarrea, que fue el efecto adverso más común asociado con el tegaserod, solía ser de corta duración y ocurrió en el 9-10\% de los pacientes que recibieron el fármaco, frente al 4-5\% de los que tomaron placebo. La diarrea fue la causa de suspensión del tratamiento con tegaserod en el 1 al $2 \%$ de los pacientes ${ }^{118-123}$.

La experiencia latinoamericana con el tegaserod se ha comunicado recientemente en un estudio multinacional abierto de 678 pacientes (92\% mujeres) que presentaban SII con predominio de estreñimiento ${ }^{125}$. Todos los pacientes recibieron $12 \mathrm{mg} /$ día de tegaserod durante 4 semanas. Se aleatorizó a los pacientes que respondieron para continuar con el mismo tratamiento durante 8 semanas más o para suspenderlo. Se evaluaron la respuesta global, los síntomas individuales de SII y la recurrencia de síntomas al suspender el tratamiento. El 82\% de los pacientes respondieron al tratamiento inicial de 4 semanas y el $90 \%$ al final de las 12 semanas. La recidiva de síntomas se observó en el $67 \%$ de los que habían respondido inicialmente al tratamiento con tegaserod y luego lo suspendieron, y sólo en el $10 \%$ de los que continuaron con el fármaco. La 
recidiva de los síntomas ocurrió, en promedio, a los 21 días de haber suspendido el tegaserod. La cefalea (15\%) y la diarrea (9\%) fueron los efectos adversos más frecuentemente observados en este estudio ${ }^{125}$.

En resumen, el tegaserod, el agonista parcial de los receptores $5 \mathrm{HT}_{4}$, es efectivo y seguro en pacientes con SII con predominio de estreñimiento. A dosis de $6 \mathrm{mg} 2$ veces al día produce mejoría global de los síntomas de SII, alivia el dolor o malestar abdominales, la distensión y la frecuencia y consistencia de las evacuaciones (recomendación de grado A).

\section{PSICOTERAPIA (INCLUIDA HIPNOTERAPIA) Y TRATAMIENTO CONDUCTUAL}

La relación de los trastornos psicológicos y el SII es ampliamente reconocida. Se ha comunicado que mediante el uso de entrevistas especializadas se detectan trastornos psiquiátricos en aproximadamente el $12 \%$ de la población que acude a una consulta general, pero que este porcentaje es de alrededor del 35\% de la población remitida a los especialistas y alcanza al 90\% de los enfermos que no responden al tratamiento ${ }^{126}$. Por otra parte, los pacientes con SII relatan frecuentemente que el estrés psicológico se asoció al inicio o produce exacerbación de los síntomas digestivos. En otros casos, los pacientes refieren historias de traumas emocionales, como son el maltrato físico, la violencia intrafamiliar, el abuso sexual, la muerte de un ser querido, la separación o el divorcio de los padres, la falta de adaptación a situaciones ambientales y otras situaciones que ocurrieron durante la niñez o adolescencia ${ }^{127}$. Es importante reconocer esta asociación porque a menudo algunos pacientes con SII con síntomas de moderados a graves y con trastornos psicológicos o psiquiátricos concomitantes son los que más consultan y en quienes las medidas sintomáticas convencionales son menos eficaces.

La necesidad y la indicación del tratamiento psicológico dependen, obviamente, de la gravedad del trastorno psicológico o de la enfermedad psiquiátrica concomitante, lo que puede evaluar, en cierto modo, el médico de atención primaria, quien puede determinar la necesidad de remitir al especialista en el plan global de tratamiento.

En los casos leves puede bastar con que el médico de atención primaria precise esta relación, asegure al paciente el diagnóstico de SII y recomiende medidas simples que permitan a éste controlar el estrés. El perfil psicológico de estos pacientes no difiere significativamente del de la población control.

Aproximadamente otro 25\% de los pacientes con SII tienen síntomas más graves, el perfil psicológico está más alterado y en ellos está indicada la interconsulta para determinar la psicoterapia y eventualmente algunos fármacos como sedantes o antidepresivos. Por último, resta aproximadamente otro 5\% de pacientes con síntomas psiquiátricos francos, quienes además presentan los síntomas digestivos más graves y rebeldes. Los trastornos más frecuentes son la ansiedad o depresión graves, que a menudo precisan de intervención psiquiátrica, incluida la farmacoterapia $^{128}$.
Las terapias psicológicas más utilizadas son la cognitiva, la conductual, la dinámica multicomponente, con técnicas de manejo del estrés e hipnosis. La evaluación de los resultados con el uso de estas diversas técnicas ha resultado difícil por problemas metodológicos. Las publicaciones incluyen a pacientes con SII sin un criterio bien definido, como es el de Roma II, el número de pacientes es insuficiente, no hay un grupo control y no es posible tener una evaluación «ciega» de los resultados ${ }^{129,130}$. En Latinoamérica este problema se ha visto agravado porque las escuelas psicoterapéuticas consideran a cada paciente como una unidad no comparable con otros. A todo lo anterior hay que agregar que estos tratamientos, especialmente cuando son prolongados, tienen un coste alto y no siempre se completan, lo que impide evaluar su efectividad científicamente.

Recientemente, se están haciendo estudios más sistemáticos con el uso de las técnicas de psicoterapia que arrojan resultados positivos. Por ejemplo, Drossman et $\mathrm{al}^{131} \mathrm{co}-$ municaron el resultado de un estudio multicéntrico aleatorizado en el que se compararon la terapia cognitiva con la educación en 215 pacientes con síntomas de moderados a graves de SII. Los autores observaron que la terapia cognitiva durante 12 semanas fue significativamente más efectiva que la educación sola, con tasas positivas de respuesta del 70 frente al 37\%, respectivamente.

La hipnoterapia es otra técnica que se ha utilizado en el abordaje del SII y que posiblemente altere el proceso del dolor en el $\mathrm{SNC}^{132}$. Se estima que la mayoría de los pacientes con SII pueden ser hipnotizados, pero es necesario tener su consentimiento. Los ensayos clínicos no han sido concluyentes, aunque recientemente Gonzalkorale et $\mathrm{al}^{133}$, pioneros en este tema, comunicaron una experiencia en 250 pacientes con SII sometidos a hipnoterapia en 12 sesiones durante 3 meses. Los autores observaron que esta terapia produjo mejoría significativa en estos pacientes, aunque los resultados no fueron significativos en una subpoblación de varones con SII y predominio de diarrea.

La terapia cognitiva tiene recomendación de grado $A$.

Como conclusión, los tratamientos psicológicos y la hipnoterapia pueden recomendarse especialmente en pacientes con SII refractarios al tratamiento farmacológico ( $r e-$ comendación de grado B).

\section{TRATAMIENTO NO CONVENCIONAL}

El éxito limitado de los tratamientos médicos tradicionales en el SII ha dado como resultado que muchos pacientes busquen alternativas.

\section{Probióticos}

La microflora fecal en el SII está conformada por una alta concentración de organismos facultativos y una concentración baja de lactobacilos y bifidobacterias. Dada la patogenia del SII, en la que parecen intervenir factores inflamatorios, infecciosos o incluso relacionados con la población bacteriana, hay fundamento para considerar los 
probióticos como una posibilidad terapéutica en estos pacientes. Probablemente no todos los probióticos tienen la misma función ni son efectivos en el mismo tipo de enfermedades, ya que existen cepas específicas que pueden ser beneficiosas para el tratamiento de distintas entidades, entre ellas el SII.

Lactobacillus plantarum, con conocidas propiedades probióticas, se utilizó en un estudio aleatorizado y doble ciego, donde se demostró que disminuía significativamente el dolor y la flatulencia en pacientes con SII frente a aquellos a quienes se les administró un placebo.

Todavía son pocos los estudios realizados al respecto y serán necesarios más datos antes de sacar conclusiones definitivas, pero las perspectivas son esperanzadoras ${ }^{133,134}$.

\section{Agonistas opiáceos de los receptores kappa}

La hipersensibilidad visceral parece ser un factor importante en la patogenia del SII, por lo que se está estudiando el efecto de diversos agentes farmacológicos con una posible acción antinociceptiva. La fedotozina es el exponente de este grupo de fármacos y se ha estudiado en pacientes con $\mathrm{SII}^{135,136}$.

\section{Somatostatina y análogos de la somatostatina}

La somatostatina y sus análogos tienen un efecto analgésico del dolor somático y visceral que se debe a un bloqueo en la transmisión de la información aferente nociceptiva en la médula. El octreótido, un análogo sintético de la somatostatina, reduce la secreción gastrointestinal, el tiempo de tránsito orocecal y la sensibilidad intestinal. Sin embargo, la vía de administración (subcutánea) y su coste limitan su eventual aplicación en el tratamiento del SII.

\section{Agonistas de la hormona liberadora de gonadotropina}

La leuprorelina es un agonista de la hormona liberadora de gonadotropina que actúa disminuyendo la concentración de hormonas sexuales en la sangre. A partir de la observación de que el SII es más frecuente en mujeres y de que además los síntomas empeoran en el período postovulatorio (hechos éstos atribuidos a los efectos miorrelajantes sobre la fibra muscular lisa de la progesterona), un grupo de investigadores utilizó la leuprorelina en un estudio que comparaba este fármaco con placebo en pacientes con SII. Los resultados de este estudio mostraban una mejoría significativa de los síntomas cuando se utilizaba la leuprorelina, que se mantenía en el tiempo mientras se administraba el fármaco ${ }^{137}$. No obstante, el hecho de que algunas mujeres quedasen amenorreicas y algunos varones impotentes limita enormemente el uso clínico de la leuprorelina (recomendación de grado $C$ ).

\section{Antagonistas de la colecistocinina}

La loxiglumida, un antagonista de la colecistoquinina, reduce el tiempo de tránsito colónico y podría tener efectos beneficiosos en pacientes con SII, aunque todavía no hay estudios amplios que demuestren este hecho (recomendación de grado $C)^{138}$.

\section{Acupuntura}

La acupuntura no tiene un efecto terapéutico específico en el SII (recomendación de grado $C$ ).

\section{Hierbas medicinales}

Los tratamientos del SII con preparados de hierbas medicinales se han usado durante muchos años sin una evidencia que los apoye, salvo un estudio controlado con placebo que demostró que la administración de un preparado de hierbas chinas produjo alivio significativo de los síntomas y mejoró la calidad de vida. La compleja composición del preparado impide hacer una recomendación específica $^{139}$.

\section{Extracto de menta}

Este aceite, extraído de la Mentha X piperita L., contiene entre sus principales componentes el mentol, que posee una actividad y un perfil farmacológicos similares a los de los antagonistas del calcio; de ahí que, debido a su efecto relajante sobre el músculo liso gastrointestinal, se haya probado para aliviar los síntomas del SII. Sin embargo, su utilidad está lejos de ser establecida todavía en el tratamiento del $\mathrm{SII}^{140}$.

\section{RESUMEN Y CONCLUSIONES}

Si bien hay numerosas opciones terapéuticas en el abordaje del SII, la mayoría de los tratamientos usados están basados en tradiciones y suposiciones no sustentadas por estudios de nivel I o II. Muchas de las terapias revisadas en este consenso están dirigidas al manejo de un solo síntoma, por ejemplo, anticolinérgicos para el dolor, loperamida para la diarrea o fibras para el estreñimiento. Muchos de estos fármacos tienen un efecto que no es superior a placebo, se asocian a efectos secundarios cuya gravedad limita su uso o bien no están suficientemente evaluados.

Una nueva era de estudios de nivel I con compuestos agonistas o antagonistas de la serotonina ha permitido orientar más científicamente el tratamiento del SII. De estos estudios se desprende que en pacientes con SII de moderado a grave y con predominio de diarrea, que no responden a medidas terapéuticas convencionales puede indicarse el alosetrón, siempre que se haga hincapié en los posibles efectos adversos, que son el estreñimiento y 
eventualmente la colitis isquémica (recomendación de grado $A)$.

Por otro lado, de acuerdo con ensayos de nivel de evidencia I, especialmente en pacientes de sexo femenino que presentan SII con predominio de estreñimiento, es recomendable indicar el tegaserod (recomendación de grado A).

\section{AGRADECIMIENTOS}

Los autores agradecen a Melanie Elder, de Novartis, su entusiasta apoyo durante el desarrollo de este trabajo y a Silvia de Goyeneche su asistencia en la preparación del manuscrito.

\section{BIBLIOGRAFÍA}

1. Evidence-Based Position Statement on the Management of Irritable Bowel Syndrome in North America. Amer J Gastroenterol 2002;97:S1-S5.

2. Manning AP, Thompson WG, Heaton KW, et al. Towards a positive diagnosis of the irritable bowel. Br Med J 1978;2: 653-4.

3. Thompson WG, Longstreth GF, Drossman DA, et al. Functional bowel disorders and functional abdominal pain. Gut 1999; 45(Suppl 2):43-7.

4. Vanner SJ, Depew WT, Paterson W, Lacosta LR, Groll AG, Simon JB, et al. Predictive value of the Roma criteria for diagnosing the irritable bowel syndrome. Am J Gastroenterol 1999;94:2912-7.

5. Thompson WG. Irritable bowel syndrome: prevalence, prognosis and consequences. CMAJ 1986;134:111-3.

6. Longstreth GF, Wolde-Tsadik G. Irritable bowel-type symptoms in HMO examinees: prevalence, demographics and clinical correlates. Dig Dis Sci 1993;38:1581-9.

7. Bommelaer G, Rouch M, Dapoigny M, Pais D, Loisy P, Gaylino M, et al. Epidemiology of functional bowel disorders in apparently healthy people. Gastroenterol Clin Biol 1986;10:7-12.

8. Welch GW, Pomare EW. Functional gastrointestinal symptoms in a Wellington community sample. NZ Med J 1990; 103:418-20.

9. Kay L, Jorgensen T, Jensen KH. The epidemiology of irritable bowel syndrome in a random population: prevalence, incidence, natural history and risk factors. J Intern Med 1994;236: 23-30.

10. Bi-Zhen W, Qi-Ying P. Functional bowel disorders in apparently healthy Chinese people. Chinese J Epidemiol 1988;9: 345-9.

11. Drossman DA, Li Z, Andruzzi E, et al. US householder survey of functional gastrointestinal disorders: prevalence, sociodemography and health impact. Dig Dis Sci 1993;38:1569-80.

12. Zuckerman MJ, Guerra LG, Drossman DA, Foland JA, Gregory GG. Comparison of bowel patterns in Hispanics and non-Hispanic whites. Dig Dis Sci 1995;40:1761-9.

13. Hungin APS, Whorwell PJ, Tack J, Mearin F. The prevalence, patterns and impact of irritable bowel syndrome: an international survey of 40.000 subjects. Aliment Pharmacol Ther 2003; 17:643-50.

14. Mitchell CM, Drossman DA. Survey of the AGA membership relating to patients with functional gastrointestinal disorders. Gastroenterology 1987;92:1282-4.

15. Talley NJ, Zinsmeister AR, Van Dyke C, Melton LJ III. Epidemiology of colonic symptoms and the irritable bowel syndrome. Gastroenterology 1991;101:927-34.

16. Jones R, Lydeard S. Irritable bowel syndrome in the general population. BMJ 1992;304:87-90.

17. Mendis BLJ, Wijesiriwardena BC, Sheriff JHR, Dharmadasa K. Irritable bowel syndrome. Ceylon Med J 1982;27:171-81.

18. Sobral DT, Vidigal KS, Farias e Silva K. Síntomas digestivos em jovens: inquérito em estudantes de medicina. Arq Gastroenterol (Sao Paolo) 1991;28:27-32.
19. Quilici FA, André SB. Um consenso nacional: síndrome do intestino irritável. Sao Paulo: Lemos Editorial, 2000.

20. Ángel LA, Amaya A, Perilla C, Góngora A, Sánchez A, Bohórquez C, et al. Prevalencia del síndrome de intestino irritable y factores asociados. Acta Med Col 1997;22:219-24.

21. Huerta I, Valdovinos MA, Schmulson M. Irritable bowel syndrome in México. Dig Dis 2001;19:251-7.

22. Tanimoto MA, Schmulson M, Ramírez D, Valdovinos MA. Prevalencia de trastornos funcionales digestivos en estudiantes de medicina. Rev Gastroenterol Mex 1995;60(Suppl 3):57.

23. Toma R, Iade B. Frecuencia del síndrome de intestino irritable en una población de Montevideo. Montevideo: Hospital de Clínicas «Dr. Manuel Quintela», Escuela de Graduados, Clínica de Nutrición y Digestivo Profesor Dr. Elbio Zevallos, agosto de 2000.

24. Schmulson MJ, Valdovinos MA. Utilización de recursos médicos por los pacientes con síndrome de intestino irritable en un hospital de tercer nivel. Rev Gastroenterol Mex 1998;63:6-10.

25. Schmulson M, Remes JM, Olivas T, Valdovinos MA, Hinojosa C, Chang L, et al. Clinical characteristics and QOL in IBS patients from Mexico and the USA: are they different? Gastroenterology 2003;124(Suppl 1):A395.

26. Fieling JF. Surgery and the irritable bowel syndrome: the singer as well as the song. J Ir Med 1988;76:33-4.

27. Remes JM, Valdovinos MA, Hinojosa C, Schmulson MJ. IBS patients differ according to age: self-reported symptom severity is related. Am J Gastroenterol 2003;98:5268.

28. Francisconi CF, Meneghelli U, Quilici F, Haddad MT, André $\mathrm{S}$. A comparison of how gastroenterologists (GE) and internists (INT) evaluate patients with irritable bowel syndrome (IBS). Gut 1999;45(Suppl 5):A340.

29. Bojórquez MC, Larrosa A, Martínez EO. Dolor abdominal crónico (DAC) en niños: ¿síndrome de intestino irritable (SII) versus dispepsia no ulcerosa (DNU)? Rev Gastroenterol Méx 2000;65(resúmenes):A52.

30. Drossman DA, Whitehead WE, Camilleri M. Irritable bowel syndrome: a technical review for practice guideline development. Gastroenterology 1997;112:2120-37.

31. Mertz H. Review article: visceral hypersensitivity. Aliment Pharmacol Ther 2003;17:623-33.

32. Mertz H. Altered CNS processing of visceral pain in IBS. En: Camilleri M, Spiller RC, editors. Irritable bowel syndrome diagnosis and treatment. London: WB Saunders, 2002; p. 55-68.

33. Camilleri M, Choi M-G. Irritable bowel syndrome. Aliment Pharmacol Ther 1997;11:3-15.

34. Silverman DHS, Munakata JA, Ennes H, et al. Regional cerebral activity in normal and pathologic perception of visceral pain. Gastroenterology 1997;112:64-72.

35. Quera R, Valenzuela J. Hipersensibilidad visceral: un concepto a nuestro alcance. Rev Med Chile 2003;131:85-92.

36. Schmulson MJ, Valdovinos MA, Milke P. Chili pepper and rectal hyperalgesia in irritable bowel syndrome. Am J Gastroenterol 2003;98:1214-5.

37. Serra J, Azpiroz F, Malagelada JR. Impaired transit and tolerance of intestinal gas in the irritable bowel syndrome. Gut 2001;48:14-9.

38. Spiller RC. Postinfectious irritable bowel syndrome. Gastroenterology 2003;124:1662-71.

39. Drossman DA. Psychosocial aspects of the functional gastrointestinal disorders. En: Corazziari E, editor. Neurogastroenterology. Berlin: Walter de Gruyter, 1996; p. 33-40.

40. Drossman DA, Leserman J, Nachman G, et al. Sexual and physical abuse in women with functional or organic gastrointestinal disorders. Ann Intern Med 1990;113:828-33.

41. Talley NJ, Fett SL, Zinsmeister AR, Melton LJ III. Gastrointestinal tract symptoms and self-report abuse: a population based study. Gastroenterology 1994;107:1040-9.

42. Whitehead WE, Palsson OS. Is pain sensitivity a biological marker for irritable bowel syndrome: psychological influences on pain perception. Gastroenterology 1998;115:1263-71.

43. Creed F. Psychopatology of the functional disorders of the gut. En: Philips S, Wingate DL, editors. Functional disorders of the gut. London: Churchill \& Livingstone, 1998.

44. Hahn BA, Kirchdoerfer LJ, Fullerton S, et al. Patient-perceived severity of irritable bowel syndrome in relation to symptoms, health resource utilization, and quality of life. Aliment 
Pharmacol Ther 1997;11:553-9.

45. Yacavone RF, Locke GR III, Provenzale DT, Eisen GM. Quality of Life measurement in gastroenterology: what is available? Am J Gastroenterol 2001;96:285-97.

46. Schmulson M, Lee OY, Chang L, et al. Symptom differences in moderate to severe IBS patients based on predominant bowel habit. Am J Gastroenterol 1999;94:2929-35.

47. Testa MA, Simonson DC. Assessment of quality of life outcomes. N Engl J Med 1966;334:835-40.

48. Wiklund IK, Glise H. Quality of life in different gastrointestinal conditions. Eur J Surg Suppl 1998;582:56-61.

49. Steward AL, Greenfield S, Hays RD, et al. Functional status and well being of patients with chronic conditions: results from the medical outcomes study. JAMA 1989;262:907-13

50. Moraes-Filho JPP. Doenças funcionais do aparelho digestivo: a importância 2 distúrbios emocionais na gênese dos sintomas. Rev Bras Med 1994;51:1395-404.

51. El-Serag HB. Impact of irritable bowel syndrome: prevalence and effect on health-related quality of life. Rev Gastroenterol Dis 2003;3(Suppl 2):3-11.

52. El-Serag HB, Olden K, Bjorkman D. Health-related quality of life among persons with irritable bowel syndrome: a systematic review. Aliment Pharmacol Ther 2002;16:1171-85.

53. Drossman DA, Whitehead WE, Toner BB, et al. What determines severity among patients with painful functional bowel disorders? Am J Gastroenterol 2000;95:974-80.

54. Wells NE, Hahn BA, Whorwell PJ. Clinical economical review: irritable bowel syndrome. Aliment Pharmacol Ther 1997; 11:1019-30.

55. Patel PP, Petita A, Fogel R, et al. The economic impact of irritable bowel syndrome in a managed care setting. J Clin Gastroenterol 2000;35:14-20.

56. Talley NJ, Philips SF, Melton LJ, et al. Diagnostic value of the Manning criteria in irritable bowel syndrome. Gut 1990; 31:77.

57. Schmulson MW, Chang L. Diagnostic approach to the patient with irritable bowel syndrome. Am J Med 1999;107:205.

58. Thompson WG, Longstreth G, Drossman DA, et al. En: Rome II: the functional gastrointestinal disorders. 2nd ed. p. 335408.

59. Lynn RB, Friedman LS. Irritable bowel syndrome. N Engl J Med 1993;329:1940

60. Swarbrick ET, Bat L, Hegarty JE, et al. Site of pain from the irritable bowel. Lancet 1980;2:443.

61. Connell AM, Hilton C, Irvine G, et al. Variation of bowel habits in two population samples. Br Med J 1965;2:1095.

62. Whorwell PJ, McCallum M, Creed FH, Roberts CT. Non-colonic features of irritable bowel syndrome. Gut 1986;27:37-40.

63. White AM, Stevens WH, Upton AR, et al. Airway responsiveness to inhaled metacholine in patients with irritable bowel syndrome. Gastroenterology 1991;100:68-74.

64. Hudson JI, Goldenberg DL, Pope HG, et al. Comorbidity of fibromyalgia with medical and psyquiatric disorders. Am J Med 1992;92:363.

65. Vanner SJ, Depew WT, Paterson WG, et al. Predictive value of the Rome criteria for diagnosing the irritable bowel syndrome. Am J Gastroenterol 1999;94:2912.

66. Brandt LJ, Bjorkman D, Fennerty MB, et al. Systematic review on the management of irritable bowel syndrome in North America. Am J Gastroenterol 2002;97:S7-S26.

67. Drossman DA, Whitehead WE, Camillieri M. Irritable bowel syndrome: a technical review for practice guideline development. Gastroenterology 1997;112:2120-37.

68. Curioso W, Mendoza D. Prevalencia y asociación de la dispepsia y el síndrome de intestino irritable en una comunidad de la selva peruana. Rev Gastroent Perú 2002;22:129-40.

69. Katon WJ, Von Korff M, Lin E. Panic disorder: relationship to high medical utilization. Am J Med 1992;92:7S

70. Drossman DA, Thompson WG. The irritable bowel syndrome: review and a graduated multi-component treatment approach. Ann Intern Med 1992;116:1009.

71. Awad RA, Cordova VH, Dibildox M, et al. Reduction of post prandial motility by pinaverium bromide a calcium channel blocker acting selectively on the gastrointestinal tract in patients with irritable bowel syndrome. Acta Latinoamer Gastroenterol 1997;27:247-51.

72. Bulat R, Caldarelli C, Tougas G. Therapeutic utility of antis- pasmodics in IBS. En: Camilleri M, Spiller R, editors. Irritable bowel syndrome diagnosis and treatment. Edimburgh: Elsevier Science Limited, 2002; p. 173-8.

73. Awad R, Dibildox M, Ortiz F. The irritable bowel syndrome treatment using pinaverium bromide as a calcium channel blocker. A randomized, double blind placebo-controlled trial. Acta Gastroenterol Latinoam 1995;25:137-44.

74. Poynard T, Naveau S, Mory B, Chaput JC. Meta-analysis of smooth muscle relaxants in the treatment of irritable bowel syndrome. Aliment Pharmacol Ther 1994;8:499-510.

75. Poynard T, Regimbeau C, Benhamou Y. Meta-analysis of smooth muscle relevants in the treatment of irritable bowel syndrome. Aliment Pharm Ther 2001;15:355-61.

76. Jailwala J, Imperiale T, Kroenke K. Pharmacologic management of IBS. A systematic review of randomized controlled trials. Ann Intern Med 2000;133:136-47.

77. Veldhuyzen van Zante SJO, Talley NJ, Bytzer P, KLein KB, Whorwell OJ, Zinsmeister AR. Design of treatment trials for functional gastroeintestinal disorders. Gut 1999;45(Suppl 2): 1169-77.

78. Akehurst R, Kaltenhaler E. Treatment of irritable bowel syndrome: review of randomized controlled trials. Gut 2001;48: 272-82.

79. Talley N. Pharmacologic therapy for the irritable bowel syndrome. Am J Gastroenterol 2003;98:750-8.

80. Burkitt DP, Walker ARP, Painter NS. Effect of dietary fiber on stools and transit times and its role in the causation of disease. Lancet 1971;2:1408-11.

81. Bright-See E, MacKeown-Eysson GE. Estimation of per capita crude and dietary fiber supply in 38 countries. Am J Clin Nutr 1984;39:821-9.

82. Vincent R, Roberts A, Frier M. Effect of bran particle size on gastric emptying and bowel transit in humans: a scintigrafic study. Gut 1995;37:216-9.

83. MacIntyre A, Vincent RM, Perkins AC, Spiller RC. Effect of bran, ispaghula and inert plastic particles on gastric emptying and small bowel transit in humans. Gut 1997;40:223-7.

84. Longstreth GF, Fox DD, Youkeles L, Forsythe AB, Wolochow DA. Psyllium therapy in the irritable bowel syndrome. A double-blind trial. Ann Intern Med 1981;95:53-6.

85. Tomás M, Anon R, Mínguez M. The efficacy of Platago ova$t a$ as a regulator of intestinal transit. A double-blind study compared to placebo. Rev Esp Enferm Dig 1992;82:511-4.

86. Arffmann S, Andersen JR, Hegnhoj J, Schaffalitzky DMO, Mogensen NB. The effect of coarse wheat bran in the irritable bowel syndrome. A double-blind crossover study. Scand J Gastroenterol 1985;20:295-8.

87. Snook J, Shepherd HA. Bran supplementation in the treatment of the irritable bowel syndrome. Aliment Pharmacol Ther 1994; 8:511-4.

88. Lucey MR, Clark ML, Lowndes J, Dawson AM. Is bran efficacious in the irritable bowel syndrome? A double blind placebo controlled crossover study. Gut 1987;28:221-5.

89. Mortensen PB, Andersen JR, Arffmann S, Krag E. Shortchain fatty acids and the irritable bowel syndrome: the effect of wheat bran. Scand J Gastroenterol 1987;22:185-92.

90. Soltoft J, Krag B, Gudmand-Hoyer E, Kristensen E, Wulff HR. A double-blind trial of the effect of wheat bran on symptoms of the irritable bowel syndrome. Lancet 1976;1:270-2.

91. Fowlie S, Eastwood MA, Prescott R. The irritable bowel syndrome: assessment of psychological disturbance and its influence on the response to fiber supplementation. J Psychosom Res 1992;36:175-80.

92. Cook IJ, Irvine EJ, Campbell D, Shannon S, Reddy SN, Collins SM. Effect of dietary fiber on symptoms and rectosigmoid motility in patients with the irritable bowel syndrome. A controlled crossover study. Gastroenterology 1990;98:66-72.

93. Toskes PP, Connery KL, Ritchey TW. Calcium polycarbophil compared with placebo in the irritable bowel syndrome. Aliment Pharmacol Ther 1993;7:87-92.

94. Jalihal A, Kurian G. Ispaghula therapy in the irritable bowel syndrome: improvement in overall well-being is related to reduction in bowel dissatisfaction. J Gastroenterol Hepatol 1990; 5:507-13.

95. Prior A, Whorwell PJ. Double blind study of ispaghula in the irritable bowel syndrome. Gut 1987;28:1510-37.

96. Hallgren T, Fath S, Delbro DS, et al. Loperamide improves sphincter function and continence after restorative proctoco- 
lectomy. Dig Dis Sci 1994;39:2612-8.

97. Ayala E, Barúa León R. Asociación entre síndrome de intestino irritable y síndrome depresivo. Rev Gastroent Perú 2001; 21:198-204

98. Read M, Read NW, Barber DC, Duthie HL. Effects of loperamide on anal sphincter function in patients complaining of chronic diarrhea with fecal incontinence and urgency. Dig Dis Sci 1982;27:807-14.

99. Awad RA, Llorens F, Camelo AL, Sánchez M. A randomized double-blind placebo-controlled trial of lidamidin HCL in irritable bowel syndrome. Acta Gastroenterol Latinoam 2000; 30:169-75.

100. Rodríguez A, Valdez T, Llorens F. Tratamiento del colon irritable con lidamidina y psicoterapia de apoyo. Rev Gastroenterol Mex 1997;62:7-13.

101. Camilleri M, Kim D-Y, McKinzie S, et al. Clonidine in diarrea-predominant irritable bowel syndrome: a ramdomized, placebo-controlled, dose response study. Gastroenterology 2002; 122:A59.

102. Egbunike IG, Chaffeé BJ. Antidepressants in the management of chronic pain syndromes. Pharmacotherapy 1990;10:262-70.

103. Klein KB. Controlled treatment trials in the irritable bowel syndrome: a critique. Gastroenterology 1988;95:232-41.

104. Clouse RE, Lustman PJ, Geisman RA, et al. Antidepressant therapy in 138 patients with irritable bowel syndrome: a five year clinical experience. Aliment Pharmacol Ther 1994;8: 409-16.

105. Jailwala J, Imperiale PF, Kroenke K, et al. Pharmacology treatment of the irritable bowel syndrome: a systematic review of randomized controlled trials. Ann Int Med 2000;133: 136-47.

106. Jackson JL, O'Malley PG, Tomkins G, et al. Treatment of functional gastrointestinal disorders with antidepressant medications. A meta-analysis. Am J Med 2000;108:65-72.

107. American College of Gastroenterology, Functional Gastrointestinal Disorders Task Force. Evidence-based position statement on the management of irritable bowel syndrome in North America. Am J Gastroenterol 2002;97(11 suppl): $1 \mathrm{~S}-5 \mathrm{~S}$.

108. Talley N. Serotoninergic neuroenteric modulators. Lancet 2001; 358:2061-4.

109. Cubeddu LX. Serotonin mechanisms in chemoterapy-induced emesis in cancer patients. Oncology 1996;53:18-25.

110. Galligan JJ. Motility and pharmacologic therapies. Schuster atlas of gastrointestinal motiliy in health and disease. 2nd ed. BC Decker Inc. Hamilton, 2002; p. 399-410.

111. Camilleri M, Northcutt AR, Kong S. Efficacy and safety of alosetron in women with irritable bowel syndrome. Lancet 2000;355:1035.

112. Olden K, DeGarmo RG, Jhingran P, Bagby B, Decker C, Markowitz M, et al. Patient satisfaction with alosetron for the treatment of women with diarrhea-predominant irritable bowel syndrome. Amer J Gastroenterol 2002;97:3139-45.

113. Caras S, Krause G. Cilansetron shows efficacy in male and female non-constipated patients with irritable bowel syndrome. Gastroenterology 2001;120:A217.

114. Bockaert J, Fagni L, Dumuis A. $5 \mathrm{HT}_{4}$ receptors: an update. Handbook Exp Pharmacol 1997;129:439-74.

115. Fioramonti J, Million M, Bueno L. Investigations on a $5-\mathrm{HT}_{4}$ agonist (SDZ HTF 919) and its main metabolite in conscious dogs: effects on gastrointestinal motility and impaired gastric emptying [resumen]. Gastroenterology 1998;114:A752.

116. Pfannkuche H-J, Jul T, Gamse R, Hoyer D, Mattes H, Buchheit $\mathrm{K}-\mathrm{H}$. The properties of the new prokinetically active drug. SDZ HTF 919 [resumen]. Neurogastroenterol Mot 1995; 7:280.

117. Prather CM, Camilleri M, Zinsmeister AR, et al. Tegaserod accelerates orocecal transit in patients with constipation predominant irritable bowel syndrome. Gastroenterology 2000; 118:463-8.

118. Muller-Lissner S, Fumagalli I, Bardhan KD, et al. Tegaserod, a $5 \mathrm{HT}_{4}$ receptor partial agonist, relieves symptoms in irritable bowel syndrome patients with abdominal pain, bloating and constipation. Aliment Pharmacol Ther 2001;15:1655-66.

119. Lefkowitz MP, Shi Y, Schmitt C, et al. The $5 \mathrm{HT}_{4}$ partial agonist, tegaserod, improves abdominal discomfort/pain and normalizes altered bowel function in irritable bowel syndrome [resumen 396]. Am J Gastroenterol 1999;94:2676.

120. Novick J, Miner P, Krause R, Glebas K, Bliesath H, Ligozio
$\mathrm{G}$, et al. A randomized, double blind, placebo-controlled trial of tegaserod in female patients suffering from irritable bowel syndrome with constipation. Aliment Pharmacol Ther 2002; 16:1877-88.

121. Kellow J, Lee O, Chang FY, Thongsawat S, Mazlam MZ, Yuen H, et al. An Asia-Pacific, double blind, placebo controlled, randomized study to evaluate the efficacy, safety, and tolerability of tegaserod in patients with irritable bowel syndrome. Gut 2003; 52:671-6.

122. Nyhlin H, Bang C, Elsborg L, et al. Tegaserod is an effective and safe therapy for irritable bowel syndrome in a Nordic population. Gastroenterology 2003;124:A389.

123. Study \#307 Data on file. Novartis Pharmaceuticals Corporation.

124. Camilleri M. Management of the irritable bowel syndrome. Gastroenterology 2001;120:652-68.

125. The Latin America Investigation Group of Tegaserod: Uzcanga L, Cohen V. Relapse of symptoms following withdrawal of tegaserod treatment in irritable bowel syndrome with constipation (I.B.S.-C) Gastroenterology 2003;123: A571.

126. Drossman DA, Camilleri M, Mayer E, et al. AGA technical review on irritable bowel syndrome. Gastroenterology 2002; 123:2108-31.

127. Camilleri M. Management of the irritable bowel syndrome. Gastroenterology 2001;120:652-68.

128. Whitehead WE, Crowell MD, Robinson JC, et al. Effects of stressful life events on bowel symptoms: subjects with irritable bowel syndrome compared to subjects without bowel dysfunction. Gut 1992;33:825-30.

129. Brandt LJ, Bjorkman D, Fennerty MB, et al. Systematic review on the management of irritable bowel syndrome in North America. Am J Gastroenterol 2002;97(Suppl):1-26.

130. Veldhuyzen van Zanten SJO, Talley NJ, Bytzer P, et al. Design of treatment trials for functional gastrointestinal disorders. Gut 1999;45(Suppl 2):1169-77.

131. Drossman DA, Toner B, Whitehead W, et al. A multi-center randomized trial of cognitive-behavioral treatment (CBT) versus education (EDU) in moderate to severe functional bowel disorder (FBD). Gastroenterology 2003;124:A530.

132. Lea R, Houghton LA, Calvert EL, et al. Gut-focused hypnotherapy normalizes disordered rectal sensitivity in patients with irritable bowel syndrome. Aliment Pharmacol Ther 2003; 17:635-42

133. Gonzalkorale WM, Miller V, Afzal A, Whorwell PJ. Long term benefits of hypnotherapy for irritable bowel syndrome. Gut 2003;52:1623-9.

134. Quigley E, O'Mahony L, McCarthy J, Kelly P, Collins JK, Shanahan F, et al. Probiotics for the irritable bowel syndrome (IBS): a randomized, double blind, placebo-controlled comparison of lactobacillus and bifidobacterium strains. Gastroenterology 2002;122:A59.

135. O'Sullivan MA, O'Morain CA. Bacterial supplementation in the irritable bowel syndrome. A randomized double-blind placebo-controlled crossover study. Dig Liver Dis 2000;32: 302-4.

136. Read NW, Abitbol JL, Bardhan KD, Whorwell PJ, Fraitag B. Efficacy and safety of the peripheral kappa agonist fedotozine versus placebo in the treatment of functional dyspepsia. Gut 1997;41:664-8.

137. Dapoigny M, Abitbol JL, Fraitag B. Efficacy of peripheral kappa agonist fedotozine versus placebo in treatment of irritable bowel syndrome. A multicenter dose-response study. Dig Dis Sci 1995;40:2244-9.

138. Mathias JR, Clench MH, Abell TL, et al. Effect of leuprolide acetate in treatment of abdominal pain and nausea in pre menopausal women with functional bowel disease: a doubleblind, placebo-controlled, randomized study. Dig Dis Sci 1998; 43:1347-55

139. Farthing MJG. New drugs in the management of the irritable bowel syndrome. Drugs 1998;56:11-21.

140. Bensoussan A, Talley NJ, Hing M, Menzies R, Guo A, Ngu M. Treatment of irritable bowel syndrome with Chinese herbal medicine: a randomized controlled trial. JAMA 1998;280: 1585-9.

141. Pittler M, Ernst E. Peppermint for irritable bowel syndrome: a critical review and metaanalysis. Am J Gastroenterol 1998;93: 1131-5. 